\title{
Self-Similar Hyperbolicity
}

\author{
Alfonso Artigue*
}

August 29, 2018

\begin{abstract}
In this paper we consider expansive homeomorphisms of compact spaces with a hyperbolic metric presenting a self-similar behavior on stable and unstable sets. Several application are given related to Hausdorff dimension, entropy, intrinsically ergodic measures and the transitivity of expansive homeomorphisms with canonical coordinates.
\end{abstract}

\section{Introduction}

A homeomorphism $f: M \rightarrow M$ of a compact metric space $(M, \rho)$ is expansive if there is $\xi>0$ such that if $x, y \in M$ are different then $\rho\left(f^{n}(x), f^{n}(y)\right)>\xi$ for some $n \in \mathbb{Z}$. Examples of such dynamics are hyperbolic sets, in particular Anosov diffeomorphisms and basic sets of axiom A diffeomorphisms. By definition, expansivity is independent from hyperbolicity and smooth structures, it is a topological concept. However, several authors constructed special hyperbolic metrics for this kind of dynamics, see for example $[6,8,10,14,15,30,31,33]$. In this paper we will study expansive homeomorphisms with a self-similar hyperbolic metric.

To motivate our first result we recall that in [10] Fathi constructed a compatible metric, that we will denote by $\mathrm{d}_{F}$, for which $f$ is a Lipschitz isomorphism and there are $\xi>0$ and $\lambda>1$ such that $\max \left\{\mathrm{d}_{F}(f(x), f(y)), \mathrm{d}_{F}\left(f^{-1}(x), f^{-1}(y)\right)\right\} \geq$ $\lambda \mathrm{d}_{F}(x, y)$ if $\mathrm{d}_{F}(x, y) \leq \xi$. A metric as $\mathrm{d}_{F}$ is called adapted or Lyapunov hyperbolic. In [8] Dovbish obtained a hyperbolic metric $\mathrm{d}_{D}$ with an asymptotic homothetic behavior on local stable and unstable sets. That is, there are two constants $0<\lambda_{s}, \lambda_{u}<1$ such that $\mathrm{d}_{D}(f(x), f(y))$ approximates $\lambda_{s} \mathrm{~d}_{D}(x, y)$ for $y \in W_{\varepsilon}^{s}(x)$ as $\varepsilon \rightarrow 0$; with analogous estimate on local unstable sets. We recall that for $\varepsilon>0$ the local stable set of $x \in M$ is

$$
W_{\varepsilon}^{s}(x)=\left\{y \in M: \mathrm{d}\left(f^{n}(x), f^{n}(y)\right) \leq \varepsilon \text { for all } n \geq 0\right\} .
$$

The local unstable set is defined as

$$
W_{\varepsilon}^{u}(x)=\left\{y \in M: \mathrm{d}\left(f^{-n}(x), f^{-n}(y)\right) \leq \varepsilon \text { for all } n \geq 0\right\} .
$$

*Email: artigue@unorte.edu.uy. Adress: Departamento de Matemática y Estadística del Litoral, Universidad de la República, Gral. Rivera 1350, Salto, Uruguay. 
Our first result, Theorem 2.3, states that for every expansive homeomorphism of a compact metric space there are a compatible metric $\mathrm{d}$ and two constants $\xi>0$ and $\lambda>1$ such that if $\mathrm{d}(x, y)<\xi$ then

$$
\max \left\{\mathrm{d}(f(x), f(y)), \mathrm{d}\left(f^{-1}(x), f^{-1}(y)\right)\right\}=\lambda \mathrm{d}(x, y) .
$$

A hyperbolic metric with this property will be called self-similar and we say that $\xi$ is an expansive constant and that $\lambda$ is an expanding factor of the metric. Obviously, Dovbysh's conditions hold for a self-similar metric without taking limits. The construction of a self-similar hyperbolic metric that we present follows standard techniques. In fact, it is Fathi's metric with only a small variation that is explained in Remark 2.2.

In [15, Problem 2.6] Fujita, Kato and Matsumoto ask: Do Positively expansive maps expand strictly small distances? In our terminology they ask about the existence of a self-similar hyperbolic metric for a positively expansive map. A continuous map $f: M \rightarrow M$ of the compact metric space $(M, \rho)$ is positively expansive if there is $\delta>0$ such that if $x \neq y$ then $\rho\left(f^{n}(x), f^{n}(y)\right)>\delta$ for some $n \geq 0$. They give a positive answer in the case of open positively expansive maps and for positively expansive maps of graphs. In Theorem 2.8 we give a positive answer with full generality, its proof is only sketched because it is analogous to the case of expansive homeomorphisms.

The rest of the paper is devoted to explore the consequences of the selfsimilarity of a hyperbolic metric. Let us describe the content of this paper while stating more results that we obtained. In $\S 2$, besides proving Theorems 2.3 and 2.8, examples are given and basic properties of these metrics are investigated.

In $\S 3$, Theorem 3.11 , we prove the equation

$$
\operatorname{cap}(M, \mathrm{~d})=\frac{\operatorname{ent}(f)}{\log (\lambda)}
$$

relating the capacity of the space, the entropy of the homeomorphism and the expanding factor of a self-similar metric. This is a fundamental equation of self-similar hyperbolicity that holds for every expansive homeomorphism of a compact metric space with a self-similar metric. It was previously proved in [15] for positively expansive maps. For a hyperbolic metric not being self-similar only an inequality can be proved, see [10]. With this result we study the set of expanding factors. We define the ideal expanding factor as $\lambda_{\text {ideal }}=e^{\mathrm{h}(f) / \operatorname{dim}(M)}$, where dim stands for topological dimension. In Theorem 3.18 we show that if $f: M \rightarrow M$ is an expansive axiom A diffeomorphism of a compact connected manifold with self-similar metric d with ideal expanding factor on the nonwandering set then $f$ is a transitive Anosov diffeomorphism and the dimension of stable and unstable manifolds coincide.

In $\S 4$ we show that our metric at small scales looks like a max norm with respect to canonical coordinates. To explain the meaning of this statement let us recall that $f$ has canonical coordinates ${ }^{1}$ if for each $\varepsilon>0$ there is $\delta>0$ such

\footnotetext{
${ }^{1}$ In the literature an expansive homeomorphism with canonical coordinates may be called topological Anosov [1], Smale space [32], hyperbolic homeomorphism [24] and is equivalent to expansivity with the pseudo-orbit trancing property or local product structure.
} 
that $\mathrm{d}(x, y)<\delta$ implies $W_{\varepsilon}^{s}(x) \cap W_{\varepsilon}^{u}(y) \neq \emptyset$. If in addition $2 \varepsilon$ is an expansive constant of $f$ then $W_{\varepsilon}^{s}(x) \cap W_{\varepsilon}^{u}(y)$ is a singleton and we can define a map $[\cdot, \cdot]$ by

$$
W_{\varepsilon}^{s}(x) \cap W_{\varepsilon}^{u}(y)=\{[x, y]\}
$$

whenever $\mathrm{d}(x, y)<\delta$. In Theorem 4.4 we show that for all $\varepsilon>0$ there is $\delta>0$ such that if $0<\mathrm{d}(x, y)<\delta$ then

$$
\left|\frac{\max \{\mathrm{d}(x,[x, y]), \mathrm{d}([x, y], y)\}}{\mathrm{d}(x, y)}-1\right|<\varepsilon .
$$

This result is related to [8, Theorem 1.2']. In Theorem 4.6 we show that if $f$ is an expansive homeomorphism with canonical coordinates of a Peano continuum $M$ and holonomies are isometries then $f$ is transitive.

In $\S 5$ we give an application to the ergodic theory of Anosov diffeomorphisms. Using a self-similar hyperbolic metric we give a natural construction of the intrinsic measure (the probability measure with maximal entropy), also called Bowen-Margulis measure [17], of a topologically mixing expansive homeomorphism with canonical coordinates. This measure is obtained as a local product measure of Hausdorff measures on local stable and unstable sets with respect to a self-similar metric. Analogous constructions can be found in $[16,25,36]$.

I thank Mauricio Achigar, Federico Dalmao, Damián Ferraro, Ignacio Monteverde, Rafael Potrie, Armando Treibich and José Vieitez for useful conver-

sations during the preparation of this work. I thank the referee for several corrections and suggestions.

\section{Expansivity and self-similarity}

Let $(M, \rho)$ be a compact metric space and consider $f: M \rightarrow M$ a homeomorphism. We say that $f$ is expansive if there is $\xi>0$ such that if $x \neq y$ then $\rho\left(f^{n}(x), f^{n}(y)\right)>\xi$ for some $n \in \mathbb{Z}$.

Definition 2.1. Given a homeomorphism $f: M \rightarrow M$ we say that a compatible metric d on $M$ is self-similar if there are constants $\xi>0, \lambda>1$ such that if $\mathrm{d}(p, q) \leq \xi$ then

$$
\max _{|i|=1} \mathrm{~d}\left(f^{i}(p), f^{i}(q)\right)=\lambda \mathrm{d}(p, q)
$$

In this case we say that $\xi$ is an expansive constant and $\lambda$ is the expanding factor of the metric.

\subsection{Self-similar metrics for expansive homeomorphisms}

In this section we will construct a compatible self-similar metric for an arbitrary expansive homeomorphism of a compact metric space. 
Remark 2.2. A self-similar metric could be obtained following the proof of $[10 \text {, Theorem 5.1]. In doing so, we would have to change [10, Equation (16) }]^{2}$ with a sup in $n \in \mathbb{Z}$ instead of a bounded interval. However, to simplify the proof of Theorem 2.3 we will start assuming Fathi's metric.

As usual, the diameter of a set with respect to a metric $\rho$ is defined as

$$
\operatorname{diam}_{\rho}(A)=\sup _{x, y \in A} \rho(x, y)
$$

for all $A \subset M$.

Theorem 2.3. Every expansive homeomorphism $f: M \rightarrow M$ on a compact metric space admits a self-similar metric.

Proof. We start considering from [10, Theorem 5.1] an adapted hyperbolic metric $\mathrm{d}_{F}$ making $f$ and $f^{-1}$ Lipschitz. That is, there are $\xi_{F}>0$ and $k \geq \lambda>1$ such that

$$
k \mathrm{~d}_{F}(x, y) \geq \max _{|i|=1} \mathrm{~d}_{F}\left(f^{i}(x), f^{i}(y)\right)
$$

for all $x, y \in M$ and

$$
\max _{|i|=1} \mathrm{~d}_{F}\left(f^{i}(x), f^{i}(y)\right) \geq \lambda \mathrm{d}_{F}(x, y)
$$

if $\mathrm{d}_{F}(x, y) \leq \xi_{F}$. Consider the metric $\mathrm{d}: M \times M \rightarrow \mathbb{R}$ defined as

$$
\mathrm{d}(x, y)=\max _{i \in \mathbb{Z}} \frac{\mathrm{d}_{F}\left(f^{i}(x), f^{i}(y)\right)}{\lambda^{|i|}} .
$$

Since $M$ is compact the distances are bounded, which implies that (3) is a maximum and $d$ is a metric. Note that $d \geq d_{F}$. To prove that the metrics $d_{F}$ and d define the same topology on $M$ it only remains to show that for all $\varepsilon>0$ there is $\delta>0$ such that if $\mathrm{d}_{F}(x, y)<\delta$ then $\mathrm{d}(x, y)<\varepsilon$. Consider two different points $x, y \in M$ and take an integer $j=j(x, y) \geq 0$ such that

$$
k^{j-1}<\frac{\operatorname{diam}_{\mathrm{d}_{F}}(M)}{\mathrm{d}_{F}(x, y)} \leq k^{j} .
$$

Since

$$
\mathrm{d}_{F}\left(f^{i}(x), f^{i}(y)\right) \leq \min \left\{k^{|i|} \mathrm{d}_{F}(x, y), \operatorname{diam}_{\mathrm{d}_{F}}(M)\right\}
$$

for all $i \in \mathbb{Z}$, we have that

$$
\frac{\mathrm{d}_{F}\left(f^{i}(x), f^{i}(y)\right)}{\lambda^{|i|}} \leq \min \left\{\left(\frac{k}{\lambda}\right)^{|i|} \mathrm{d}_{F}(x, y), \frac{\operatorname{diam}_{\mathrm{d}_{F}}(M)}{\lambda^{|i|}}\right\}
$$

\footnotetext{
${ }^{2}$ For reader's convenience we point that Equation (16) is between Equations (5) and (7), see $[10$, p. 259].
} 
for all $i \in \mathbb{Z}$. Applying (4), if $|i| \leq j-1$ then

$$
\left(\frac{k}{\lambda}\right)^{|i|} \mathrm{d}_{F}(x, y) \leq\left(\frac{k}{\lambda}\right)^{j-1} \mathrm{~d}_{F}(x, y) \leq \frac{\operatorname{diam}_{\mathrm{d}_{F}}(M)}{\lambda^{j-1}} .
$$

For $|i| \geq j$ it holds that $\operatorname{diam}_{\mathrm{d}_{F}}(M) / \lambda^{|i|} \leq \operatorname{diam}_{\mathrm{d}_{F}}(M) / \lambda^{j}$. Then

$$
\min \left\{(k / \lambda)^{|i|} \mathrm{d}_{F}(x, y), \operatorname{diam}_{\mathrm{d}_{F}}(M) / \lambda^{|i|}\right\} \leq \operatorname{diam}_{\mathrm{d}_{F}}(M) / \lambda^{j-1}
$$

for all $i \in \mathbb{Z}$. Applying (5) we obtain

$$
\mathrm{d}(x, y)=\max _{i \in \mathbb{Z}} \frac{\mathrm{d}_{F}\left(f^{i}(x), f^{i}(y)\right)}{\lambda^{|i|}} \leq \frac{k \operatorname{diam}_{\mathrm{d}_{F}}(M)}{\lambda^{j-1}} .
$$

Since $j(x, y) \rightarrow+\infty$ as $\mathrm{d}_{F}(x, y) \rightarrow 0$ the metrics $\mathrm{d}$ and $\mathrm{d}_{F}$ are compatible.

To prove (2) take $\xi>0$ such that if $\mathrm{d}(x, y)<\xi$ then $\mathrm{d}_{F}(x, y)<\xi_{F}$. We have that

$$
\begin{aligned}
\operatorname{maxd}\left(f^{ \pm 1}(x), f^{ \pm 1}(y)\right) & =\max _{i \in \mathbb{Z}} \frac{\mathrm{d}_{F}\left(f^{i \pm 1}(x), f^{i \pm 1}(y)\right)}{\lambda^{|i|}} \\
& =\max _{i \in \mathbb{Z}} \frac{\mathrm{d}_{F}\left(f^{i}(x), f^{i}(y)\right)}{\lambda^{|i \pm 1|}}
\end{aligned}
$$

Since $\min \{|i+1|,|i-1|\} \geq|i|-1$ for all $i \in \mathbb{Z}$ we conclude

$$
\max _{i \in \mathbb{Z}} \frac{\mathrm{d}_{F}\left(f^{i}(x), f^{i}(y)\right)}{\lambda^{|i \pm 1|}} \leq \max _{i \in \mathbb{Z}} \frac{\mathrm{d}_{F}\left(f^{i}(x), f^{i}(y)\right)}{\lambda^{|i|-1}}
$$

Notice that the difference between $\min |i \pm 1|$ and $|i|-1$, for an integer $i$, is only at $i=0$, where $\min |0 \pm 1|=1$ and $|0|-1=-1$. We know that if $\mathrm{d}(x, y)<\xi$ then $\mathrm{d}_{F}(x, y)<\xi_{F}$ and consequently

$$
\max \mathrm{d}_{F}\left(f^{ \pm 1}(x), f^{ \pm 1}(y)\right) \geq \lambda \mathrm{d}_{F}(x, y) .
$$

Then

$$
\max _{i \in \mathbb{Z}} \frac{\mathrm{d}_{F}\left(f^{i}(x), f^{i}(y)\right)}{\lambda^{|i \pm 1|}} \geq \max _{|i|=1} \frac{\mathrm{d}_{F}\left(f^{i}(x), f^{i}(y)\right)}{\lambda^{|i \pm 1|}} \geq \lambda \mathrm{d}_{F}(x, y) .
$$

This proves that

$$
\max _{i \in \mathbb{Z}} \frac{\mathrm{d}_{F}\left(f^{i}(x), f^{i}(y)\right)}{\lambda^{|i \pm 1|}}=\max _{i \in \mathbb{Z}} \frac{\mathrm{d}_{F}\left(f^{i}(x), f^{i}(y)\right)}{\lambda^{|i|-1}}
$$

Finally

$$
\max _{i \in \mathbb{Z}} \frac{\mathrm{d}_{F}\left(f^{i}(x), f^{i}(y)\right)}{\lambda^{|i|-1}}=\lambda \max _{i \in \mathbb{Z}} \frac{\mathrm{d}_{F}\left(f^{i}(x), f^{i}(y)\right)}{\lambda^{|i|}}=\lambda \mathrm{d}(x, y)
$$

which proves the result. 
Remark 2.4. From Equation (4) in the proof of Theorem 2.3 we have that

$$
\left(\frac{\operatorname{diam}_{\mathrm{d}_{F}}(M)}{\mathrm{d}_{F}(x, y)}\right)^{\log _{k}(\lambda)} \leq \lambda^{j} .
$$

Applying (6) we conclude

$$
\mathrm{d}_{F}(x, y) \leq \mathrm{d}(x, y) \leq c\left[\mathrm{~d}_{F}(x, y)\right]^{\alpha}
$$

with $\alpha=\log _{k}(\lambda) \in(0,1)$ and $c>0$. That is, $\mathrm{d}$ and $\mathrm{d}_{F}$ are Hölder equivalent, as anticipated by Fried in [14, Lemma 2].

Let us give some examples with an explicit self-similar metric.

Example 2.5 (Shifts and subshifts). Let $N^{\mathbb{Z}}$ be the space of sequences on $N$ symbols $\{0,1,2, \ldots, N-1\}$. For $a, b: \mathbb{Z} \rightarrow N$ define

$$
T(a, b)=\max \{n \geq 0: a(i)=b(i) \text { if }|i| \leq n\} .
$$

Given $\lambda>1$ define $\mathrm{d}(a, b)=\lambda^{-T(a, b)}$. It is easy to see that $\mathrm{d}$ is self-similar with respect to the shift homeomorphism $\sigma: N^{\mathbb{Z}} \rightarrow N^{\mathbb{Z}}\left(\sigma(a)_{n}=a_{n+1}\right)$. The expanding factor is $\lambda$. If $X \subset N^{\mathbb{Z}}$ is a closed $\sigma$-invariant subset (a subshift) then the restricted metric is self-similar.

Example 2.6 (Expansive homeomorphisms of surfaces). On compact surfaces we know that expansive homeomorphisms are conjugate to pseudo-Anosov diffeomorphisms, see $[18,22]$. A pseudo-Anosov diffeomorphism $f: M \rightarrow M$ of a compact surface, by definition, has two invariant singular foliations with transverse measures $\mu_{s}, \mu_{u}$ that are expanded and contracted by a factor $\lambda>1$. To define a self-similar metric, for $p, q \in M$ consider the set $C^{s u}(p, q)$ of curves $\alpha:[0,1] \rightarrow M$ from $p$ to $q$ such that there are $t=0=0<t_{1}<\cdots<t_{n}=1$ such that each $\alpha_{i}=\alpha\left(\left[t_{i}, t_{i+1}\right]\right)$ is contained in a stable or an unstable leaf of the foliations. Let $l_{s}(\alpha)$ be the sum of the $\mu_{s}$-measures of the arcs $\alpha_{i}$ contained in a stable leaf. Analogously, define $l_{u}(\alpha)$. Finally consider

$$
\mathrm{d}(p, q)=\inf _{\alpha \in C^{s u}(p, q)} \max \left\{l_{s}(\alpha), l_{u}(\alpha)\right\} .
$$

It is easy to prove that $\mathrm{d}$ is a compatible self-similar metric with expanding factor $\lambda$, where $\lambda$ is the expanding factor of the transverse measures.

We remark that Theorem 2.3 can be applied to every expansive homeomorphism of a compact metric space, in particular to Anosov diffeomorphisms of compact smooth manifolds. Under certain conditions a self-similar can be derived from a Riemannian metric.

Example 2.7 (Linear Anosov diffeomorphisms). Let $T: \mathbb{R}^{n} \rightarrow \mathbb{R}^{n}$ be a linear isomorphism inducing an Anosov automorphism $f$ of the torus $T^{n}$. Assume that the stable subspace $E^{s}$ and the unstable subspace $E^{u}$ of $\mathbb{R}^{n}$ can be writen as $E^{s}=E_{1}^{s} \oplus \cdots \oplus E_{k}^{s}$ and $E^{u}=E_{1}^{u} \oplus \cdots \oplus E_{l}^{u}$ and there are real numbers 
$0<\left|a_{1}\right|, \ldots,\left|a_{k}\right|<1,\left|b_{1}\right|, \ldots,\left|b_{l}\right|>1$ such that $T(v)=a_{i} v$ for all $v \in E_{i}^{s}$ and $T(v)=b_{j} v$ for all $v \in E_{j}^{u}$. Let $\|\cdot\|$ be a norm in $\mathbb{R}^{n}$. Given $v \in \mathbb{R}^{n}$ consider $p \in E^{s}$ and $q \in E^{u}$ such that $v=p+q$ and take $p_{i} \in E_{i}^{s}$ and $q_{j} \in E_{j}^{u}$ such that $p=p_{1}+\cdots+p_{k}$ and $q=q_{1}+\cdots+q_{l}$. For

$$
\lambda=\min \left\{\left|a_{i}\right|^{-1},\left|b_{j}\right|: 1 \leq i \leq k, 1 \leq j \leq l\right\}
$$

define

$$
\rho(v)=\max \left\{\left\|p_{i}\right\|^{\log (\lambda) \log \left|a_{i}\right|},\left\|q_{j}\right\|^{\log (\lambda) / \log \left|b_{j}\right|}: 1 \leq i \leq k, 1 \leq j \leq l\right\} .
$$

The metric $\mathrm{d}(p, q)=\rho(q-p)$ in $\mathbb{R}^{n}$ induces a self-similar metric on the torus with expanding factor $\lambda$.

\subsection{Positively expansive maps}

In this brief section we indicate how to construct a self-similar metric for a positively expansive map.

Theorem 2.8. If $f: M \rightarrow M$ is a positively expansive map of a compact metric space then there is a compatible metric $\mathrm{d}$ on $M, \xi>0$ and $\lambda>1$ such that if $\mathrm{d}(x, y)<\xi$ then $\mathrm{d}(f(x), f(y))=\lambda \mathrm{d}(x, y)$.

Proof. By [30] (see also [35]) we know that there is a compatible metric $\mathrm{d}_{R}$, $\xi>0$ and $\lambda>1$ such that if $\mathrm{d}_{R}(x, y)<\xi$ then $\mathrm{d}_{R}(f(x), f(y)) \geq \lambda \mathrm{d}_{R}(x, y)$. Also, this metric makes $f$ Lipschitz. A self-similar metric can be defined by

$$
\mathrm{d}(x, y)=\max _{n \geq 0} \frac{\mathrm{d}_{R}\left(f^{n}(x), f^{n}(y)\right)}{\lambda^{n}} .
$$

The rest of the proof is analogous to the proof of Theorem 2.3.

\subsection{Basic properties of a self-similar metric}

In the following results we investigate simple but important properties of the self-similar metric.

Remark 2.9. First note that Equation (2) implies that if $\mathrm{d}(p, q) \leq \xi$ then

$$
\begin{aligned}
& \mathrm{d}(f(p), f(q)) \leq \lambda \mathrm{d}(p, q) \text { and } \\
& \mathrm{d}\left(f^{-1}(p), f^{-1}(q)\right) \leq \lambda \mathrm{d}(p, q) .
\end{aligned}
$$

These easily gives us that $f$ and $f^{-1}$ are Lipschitz. Moreover, considering $\mathrm{d}^{\prime}(p, q)=\min \{\mathrm{d}(p, q), \xi\}$ we can assume that $\lambda$ itself is a Lipschitz constant for $f$ and $f^{-1}$. In this case the expansivity constant should be reduced to $\xi^{\prime}=\xi / \lambda$.

Proposition 2.10. If $\mathrm{d}$ is self-similar and $\mathrm{d}(f(x), f(y))=\lambda \mathrm{d}(x, y)$ then

$$
\mathrm{d}\left(f^{k}(x), f^{k}(y)\right)=\lambda^{k} \mathrm{~d}(x, y)
$$

for all $k \geq 0$ such that $\lambda^{k-1} \mathrm{~d}(x, y) \leq \xi$. 
Proof. For $k=0,1$ the result is trivial. Consider $k \geq 2$ and assume that $\mathrm{d}\left(f^{l}(x), f^{l}(y)\right)=\lambda^{l} \mathrm{~d}(x, y)$ for all $l=0,1, \ldots, k-1$. Therefore,

$$
\mathrm{d}\left(f^{k-1}(x), f^{k-1}(y)\right)=\lambda \mathrm{d}\left(f^{k-2}(x), f^{k-2}(y)\right)
$$

and, in particular, $\lambda \mathrm{d}\left(f^{k-1}(x), f^{k-1}(y)\right) \neq \mathrm{d}\left(f^{k-2}(x), f^{k-2}(y)\right)$. Since

$$
\lambda^{k-1} \mathrm{~d}(x, y)=\mathrm{d}\left(f^{k-1}(x), f^{k-1}(y)\right) \leq \xi
$$

we can apply Equation (2) to $p=f^{k-1}(x)$ and $q=f^{k-1}(y)$ to conclude $\mathrm{d}\left(f^{k}(x), f^{k}(y)\right)=\lambda \mathrm{d}\left(f^{k-1}(x), f^{k-1}(y)\right)$. Since

$$
\mathrm{d}\left(f^{k-1}(x), f^{k-1}(y)\right)=\lambda^{k-1} \mathrm{~d}(x, y)
$$

the proof ends.

Proposition 2.11. If $\mathrm{d}$ is self-similar and $\lambda \mathrm{d}(x, y) \neq \mathrm{d}\left(f^{-1}(x), f^{-1}(y)\right)$ then

$$
\mathrm{d}\left(f^{k}(x), f^{k}(y)\right)=\lambda^{k} \mathrm{~d}(x, y)
$$

for all $k \geq 0$ such that $\lambda^{k-1} \mathrm{~d}(x, y) \leq \xi$.

Proof. For $k=0$ there is nothing to prove. If $k=1$ we have $\mathrm{d}(x, y) \leq$ $\xi$. Since $\mathrm{d}(x, y) \neq \lambda^{-1} \mathrm{~d}\left(f^{-1}(x), f^{-1}(y)\right)$ we apply Equation (2) to obtain $\mathrm{d}(f(x), f(y))=\lambda \mathrm{d}(x, y)$. Then, applying Proposition 2.10 the proof ends.

Proposition 2.12. If $y \in W_{\xi}^{s}(x)$ then $\mathrm{d}\left(f^{n}(x), f^{n}(y)\right)=\lambda^{-n} \mathrm{~d}(x, y)$ for all $n \geq 0$. Analogously, if $y \in W_{\xi}^{u}(x)$ then $\mathrm{d}\left(f^{-n}(x), f^{-n}(y)\right)=\lambda^{-n} \mathrm{~d}(x, y)$ for all $n \geq 0$.

Proof. If $y \in W_{\xi}^{s}(x)$ and $\mathrm{d}\left(f^{n}(x), f^{n}(y)\right) \neq \lambda^{-n} \mathrm{~d}(x, y)$ for some $n \geq 0$ then there is $m \geq 0$ such that $\mathrm{d}\left(f^{m+1}(x), f^{m+1}(y)\right) \neq \lambda \mathrm{d}\left(f^{m}(x), f^{m}(y)\right)$. Applying Proposition 2.11 we contradict that $y \in W_{\xi}^{s}(x)$.

\subsection{Dynamical triangles}

In what follows we will assume that $f: M \rightarrow M$ is an expansive homeomorphism with a self-similar metric $\mathrm{d}$ on the compact space $M$. In addition, $\lambda>1$ and $\xi>0$ will denote the expanding factor and the expansivity constant respectively. The purpose of this section is to prove Theorem 2.21 where we will give an estimate of the metric at small scales. This Theorem will be applied to prove that a self-similar metric is a max norm at small scales assuming canonical coordinates, Theorem 4.4. However, the study of the present section does not assume canonical coordinates.

Definition 2.13. We say that $(x, y) \in M \times M$ is a critical pair if $0<\mathrm{d}(x, y)<$ $\xi, \mathrm{d}\left(f^{-1}(x), f^{-1}(y)\right)=\lambda \mathrm{d}(x, y)$ and $\mathrm{d}\left(f^{2}(x), f^{2}(y)\right)=\lambda \mathrm{d}(f(x), f(y))$. 
Note that if $(x, y)$ is a critical pair and $\mathrm{d}(f(x), f(y))<\xi$ then $(f(x), f(y))$ is a critical pair for $f^{-1}$. Fix a critical pair $(x, y) \in M \times M$ and define $c_{n}=$ $\mathrm{d}\left(f^{n}(x), f^{n}(y)\right)$ for $n \in \mathbb{Z}$. By Proposition 2.10 we have that if $n \geq 1$ then

$$
\begin{aligned}
c_{n+1}=\lambda^{n} c_{1} & \text { if } \lambda^{n-1} c_{1} \leq \xi, \\
c_{-n}=\lambda^{n} c_{0} & \text { if } \lambda^{n-1} c_{0} \leq \xi .
\end{aligned}
$$

If $c_{1}<\xi$, by Remark 2.9 we have

$$
\frac{1}{\lambda^{2}} \leq \frac{c_{1}}{\lambda c_{0}} \leq 1 .
$$

Definition 2.14. We say that $(x, y, z)$ is a dynamical triangle if $z=W_{\xi / 2 \lambda}^{u}(x) \cap$ $W_{\xi / 2 \lambda}^{s}(y)$.

Remark 2.15. For all $\varepsilon>0$ there is $\delta>0$ such that if $(x, y, z)$ is a dynamical triangle and $\mathrm{d}(x, y)<\delta$ then $\max \{\mathrm{d}(x, z), \mathrm{d}(y, z)\}<\varepsilon$. The proof is as follows. If this is not the case we can take dynamical triangles $\left(x_{n}, y_{n}, z_{n}\right)$ such that $\mathrm{d}\left(x_{n}, y_{n}\right) \rightarrow 0$ but $\mathrm{d}\left(x_{n}, z_{n}\right)$ is bounded away from zero (the other case is similar). Then, two limit points of $x_{n}$ and $z_{n}$ contradict the expansivity of $f$.

Fix a dynamical triangle $(x, y, z)$ and define $a=\mathrm{d}(x, z)$ and $b=\mathrm{d}(z, y)$. Since $a, b \leq \xi / 2 \lambda$, there are $N_{a}, N_{b}>0$ such that

$$
\begin{aligned}
& \lambda^{N_{a}} a \leq \frac{\xi}{2}<\lambda^{N_{a}+1} a, \\
& \lambda^{N_{b}} b \leq \frac{\xi}{2}<\lambda^{N_{b}+1} b .
\end{aligned}
$$

Since $z \in W_{\xi}^{u}(x)$ and $z \in W_{\xi}^{s}(x)$, from Proposition 2.12 we have that

$$
\begin{array}{ll}
\mathrm{d}\left(f^{n}(x), f^{n}(z)\right)=\lambda^{n} a & \text { if } n \leq N_{a}, \\
\mathrm{~d}\left(f^{n}(y), f^{n}(z)\right)=\lambda^{-n} b & \text { if } n \geq-N_{b} .
\end{array}
$$

From this and the triangular inequality of the metric we conclude that

$$
\left|\lambda^{n} a-\lambda^{-n} b\right| \leq c_{n} \leq \lambda^{n} a+\lambda^{-n} b
$$

whenever $-N_{b} \leq n \leq N_{a}$. By (10) we can prove that $\lambda^{N_{a}} a>\lambda^{-N_{a}} b$ and $\lambda^{-N_{b}} a<\lambda^{N_{b}} b$. Therefore, (11) with $n=N_{a}$ and $n=-N_{b}$ implies

$$
\begin{aligned}
& \lambda^{N_{a}} a-\lambda^{-N_{a}} b \leq c_{N_{a}} \leq \lambda^{N_{a}} a+\lambda^{-N_{a}} b, \\
& \lambda^{N_{b}} b-\lambda^{-N_{b}} a \leq c_{-N_{b}} \leq \lambda^{N_{b}} b+\lambda^{-N_{b}} a .
\end{aligned}
$$

Thus

$$
\frac{\lambda^{N_{a}} a-\lambda^{-N_{a}} b}{\lambda^{-N_{b}} a+\lambda^{N_{b}} b} \leq \frac{c_{N_{a}}}{c_{-N_{b}}} \leq \frac{\lambda^{N_{a}} a+\lambda^{-N_{a}} b}{\lambda^{N_{b}} b-\lambda^{-N_{b}} a} .
$$

Definition 2.16. We say that a dynamical triangle $(x, y, z)$ is a critical triangle if $(x, y)$ is a critical pair. 
Proposition 2.17. If $(x, y, z)$ is a critical triangle then

$$
\frac{a-\lambda^{-2 N_{a}} b}{b+\lambda^{-2 N_{b} a}} \leq \frac{c_{1}}{\lambda c_{0}} \leq \frac{a+\lambda^{-2 N_{a}} b}{b-\lambda^{-2 N_{b} a}} .
$$

Proof. From (10) and (11) we have that $c_{n} \leq \xi$ if $-N_{b} \leq n \leq N_{a}$. This allows us to apply (7) and (8) to obtain $c_{N_{a}}=\lambda^{N_{a}-1} c_{1}$ and $c_{-N_{b}}=\lambda^{N_{b}} c_{0}$. Now the result follows from (14).

In the following lemmas we give some estimates that we need for the proof of Theorem 2.21.

Lemma 2.18. For all $\varepsilon_{1}>0$ there is $\delta>0$ such that for any critical triangle with $c_{0}<\delta$ it holds that

$$
\frac{1}{\lambda^{2}}-\varepsilon_{1} \leq \frac{a}{b} \leq 1+\varepsilon_{1}
$$

Proof. By (9) we know that $\frac{c_{1}}{\lambda c_{0}} \leq 1$. From (15) we have $\frac{a-\lambda^{-2 N_{a}} b}{b+\lambda^{-2 N_{b} a}} \leq \frac{c_{1}}{\lambda c_{0}}$. Then $\frac{a-\lambda^{-2 N_{a}}}{b+\lambda^{-2 N_{b}}} \leq 1$. This implies that $\frac{a}{b} \leq \frac{1+\lambda^{-2 N_{a}}}{1-\lambda^{-2 N_{b}}}$. Considering again (9) and (15) we have $\frac{1}{\lambda^{2}} \leq \frac{c_{1}}{\lambda c_{0}}$ and $\frac{c_{1}}{\lambda c_{0}} \leq \frac{a+\lambda^{-2 N_{a}} b}{b-\lambda^{-2 N_{b}}}$. This gives

$$
\frac{\lambda^{-2}-\lambda^{-2 N_{a}}}{1+\lambda^{-2} \lambda^{-2 N_{b}}} \leq \frac{a}{b} \leq \frac{1+\lambda^{-2 N_{a}}}{1-\lambda^{-2 N_{b}}}
$$

By Remark 2.15, if $c_{0}$ is small then $a$ and $b$ are small. Consequently $N_{a}$ and $N_{b}$ are large. This proves (16).

Lemma 2.19. There is $\varepsilon_{0}>0$ such that for all $\varepsilon_{1} \in\left(0, \varepsilon_{0}\right)$ there is $\delta>0$ such that for any critical triangle with $c_{0}<\delta$ it holds that

$$
\frac{b}{c_{0}} \leq 2
$$

Proof. For $\varepsilon_{1}>0$ given consider $\delta>0$ from Lemma 2.18. By (13) and (8) we have $\left|c_{0}-b\right| \leq \lambda^{-2 N_{b}} a$. From (16) we conclude that $\left|1-\frac{b}{c_{0}}\right| \leq \frac{a}{c_{0}} \lambda^{-2 N_{b}} \leq$ $\frac{b\left(1+\varepsilon_{1}\right)}{c_{0}} \lambda^{-2 N_{b}}$. Then $\frac{b}{c_{0}}-1 \leq \frac{b\left(1+\varepsilon_{1}\right)}{c_{0}} \lambda^{-2 N_{b}}$ and $\frac{b}{c_{0}}\left(1-\left(1+\varepsilon_{1}\right) \lambda^{-2 N_{b}}\right) \leq 1$. Finally, take $\varepsilon_{0}>0$ such that $1-\left(1+\varepsilon_{0}\right) \lambda^{-2 N_{b}} \geq 1 / 2$.

Lemma 2.20. For all $\varepsilon_{1}>0$ there is $\delta>0$ such that if $c_{0}=\mathrm{d}(x, y)<\delta$ then

$$
\left|\frac{c_{1}}{\lambda c_{0}}-\frac{a}{b}\right|<\varepsilon_{1}
$$

Proof. Using (10) we have

$$
\frac{\lambda^{-2 N_{a}} b}{a}=\frac{b}{a \lambda^{N_{a}} \lambda^{N_{a}}} \leq \frac{2 \lambda b}{\xi \lambda^{N_{a}}} \leq \frac{\lambda}{\lambda^{N_{a}+N_{b}}} .
$$


Analogously,

$$
\frac{\lambda^{-2 N_{b}} a}{b} \leq \frac{\lambda}{\lambda^{N_{a}+N_{b}}} .
$$

Then (15), (19), (20) and (16) prove (18).

Theorem 2.21. If $\mathrm{d}$ is self-similar then for all $\varepsilon>0$ there is $\delta>0$ such that if $(x, y, z)$ is a dynamical triangle with $\operatorname{diam}(x, y, z)<\delta$ then

$$
\left|\frac{\mathrm{d}(x, y)}{\max \{\mathrm{d}(x, z), \mathrm{d}(z, y)\}}-1\right|<\varepsilon .
$$

Proof. Consider $\varepsilon_{0}>0$ from Lemma 2.19 and take $\varepsilon_{2} \in\left(0, \varepsilon_{0}\right)$. First we will show that there is $\delta>0$ such that if $(x, y, z)$ is critical and $c_{0}<\delta$ then

$$
\left|\frac{c_{0}}{\max \{a, b\}}-1\right| \leq \varepsilon_{2}
$$

For $\varepsilon_{1} \in\left(0, \varepsilon_{0}\right)$ consider $\delta>0$ satisfying Lemmas 2.18, 2.19 and 2.20. From (16) we have that $a \leq\left(1+\varepsilon_{1}\right) b$ and then

$$
b \leq \max \{a, b\} \leq\left(1+\varepsilon_{1}\right) b .
$$

Equation (12) gives $\left|c_{N_{a}}-\lambda^{N_{a}} a\right| \leq \lambda^{-N_{a}} b$. From (7) we know that $c_{N_{a}}=$ $\lambda^{N_{a}-1} c_{1}$. Therefore $\left|\frac{c_{1}}{\lambda c_{0}}-\frac{a}{c_{0}}\right| \leq \frac{b}{c_{0}} \lambda^{-2 N_{a}}$, which jointly with (18) implies $\left|\frac{a}{b}-\frac{a}{c_{0}}\right| \leq \varepsilon_{1}+\frac{b}{c_{0}} \lambda^{-2 N_{a}}$ and $\left|1-\frac{b}{c_{0}}\right| \leq \frac{b}{a}\left[\varepsilon_{1}+\frac{b}{c_{0}} \lambda^{-2 N_{a}}\right]$. By (16) we have that $\frac{b}{a} \leq \frac{\lambda^{2}}{1-\lambda^{2} \varepsilon_{1}}$. Then

$$
\left|1-\frac{b}{c_{0}}\right| \leq \frac{\lambda^{2}}{1-\lambda^{2} \varepsilon_{1}}\left[\varepsilon_{1}+\frac{b}{c_{0}} \lambda^{-2 N_{a}}\right] .
$$

This inequality and (17) gives us $\left|1-\frac{b}{c_{0}}\right| \leq \frac{\lambda^{2}}{1-\lambda^{2} \varepsilon_{1}}\left(\varepsilon_{1}+2 \lambda^{-2 N_{a}}\right)$. By (22) we have that $\left|\frac{b}{\max \{a, b\}}-1\right| \leq \frac{\varepsilon_{1}}{1+\varepsilon_{1}}$. Adding the last two inequalities and applying the triangular inequality we have $\left|\frac{b}{\max \{a, b\}}-\frac{b}{c_{0}}\right| \leq \frac{\lambda^{2}}{1-\lambda^{2} \varepsilon_{1}}\left(\varepsilon_{1}+2 \lambda^{-2 N_{a}}\right)+\frac{\varepsilon_{1}}{1+\varepsilon_{1}}$. That is $\left|\frac{c_{0}}{\max \{a, b\}}-1\right| \leq \frac{c_{0}}{b}\left[\frac{\lambda^{2}}{1-\lambda^{2} \varepsilon_{1}}\left(\varepsilon_{1}+2 \lambda^{-2 N_{a}}\right)+\frac{\varepsilon_{1}}{1+\varepsilon_{1}}\right]$. From (22) we have that

$$
\frac{\max \{a, b\}}{1+\varepsilon_{1}} \leq b
$$

and then

$$
\frac{c_{0}}{b} \leq \frac{c_{0}}{\max \{a, b\}}\left(1+\varepsilon_{1}\right) \leq 2\left(1+\varepsilon_{1}\right)
$$

because $c_{0} \leq 2 \max \{a, b\}$ (triangular inequality). Then

$$
\left|\frac{c_{0}}{\max \{a, b\}}-1\right| \leq 2\left(1+\varepsilon_{1}\right)\left[\frac{\lambda^{2}}{1-\lambda^{2} \varepsilon_{1}}\left(\varepsilon_{1}+2 \lambda^{-2 N_{a}}\right)+\frac{\varepsilon_{1}}{1+\varepsilon_{1}}\right] .
$$


From Remark 2.15 we have that if $\delta$ is small then $\lambda^{-2 N_{a}}$ is small. Then, it is clear that if $\varepsilon_{1}$ is sufficiently small then we obtain (21).

For an arbitrary dynamical triangle $\left(x^{\prime}, y^{\prime}, z^{\prime}\right)$ the proof is reduced to the case of a critical triangle as follows. Consider $\delta>0$ as before (satisfying the previous lemmas). Assume that $\operatorname{diam}\left(x^{\prime}, y^{\prime}, z^{\prime}\right)<\delta$. Suppose that for some $n>0$ the pair $\left(f^{n}\left(x^{\prime}\right), f^{n}\left(y^{\prime}\right)\right)$ is critical. If we define $x=f^{n}\left(x^{\prime}\right), y=f^{n}\left(y^{\prime}\right)$ and $z=f^{n}\left(z^{\prime}\right)$ then

$$
\begin{array}{r}
\mathrm{d}\left(x^{\prime}, y^{\prime}\right)=\lambda^{n} c_{0}, \\
\mathrm{~d}\left(y^{\prime}, z^{\prime}\right)=\lambda^{n} b, \\
\mathrm{~d}\left(x^{\prime}, z^{\prime}\right)=\lambda^{-n} a .
\end{array}
$$

In particular, $c_{0}<\delta$. By (16) we have that $\frac{a}{b}<1+\varepsilon_{1}<\lambda^{2}$ (assuming that $\left.\varepsilon_{1}<\lambda^{2}-1\right)$. Then $\max \left\{\lambda^{-2 n} a, b\right\}=b$ and

$$
\frac{\mathrm{d}\left(x^{\prime}, y^{\prime}\right)}{\max \left\{\mathrm{d}\left(x^{\prime}, z^{\prime}\right), \mathrm{d}\left(z^{\prime}, y^{\prime}\right)\right\}}=\frac{c_{0}}{\max \left\{\lambda^{-2 n} a, b\right\}}=\frac{c_{0}}{b} .
$$

From (22) we have that

$$
\frac{1}{b}-\frac{1}{\max \{a, b\}} \leq \frac{\varepsilon_{1}}{b\left(1+\varepsilon_{1}\right)}
$$

Since for the critical triangle $(x, y, z)$ we have proved $(21)$, we have

$$
\left|\frac{c_{0}}{b}-1\right| \leq\left|\frac{c_{0}}{b}-\frac{c_{0}}{\max \{a, b\}}\right|+\left|\frac{c_{0}}{\max \{a, b\}}-1\right| \leq \frac{c_{0} \varepsilon_{1}}{b\left(1+\varepsilon_{1}\right)}+\varepsilon_{2} .
$$

Jointly with (24) we obtain

$$
\left|\frac{c_{0}}{b}-1\right| \leq 2 \varepsilon_{1}+\varepsilon_{2}
$$

Finally, given $\varepsilon>0$ it is sufficient to take $\varepsilon_{1}=\varepsilon_{2}=\varepsilon / 3$.

Remark 2.22. In [32] Ruelle asked if it can be found a hyperbolic metric such that

$$
\begin{aligned}
& \mathrm{d}([x, y], x) \leq L \mathrm{~d}(x, y), \\
& \mathrm{d}([x, y], y) \leq L \mathrm{~d}(x, y) .
\end{aligned}
$$

for some $L \geq 1$, assuming expansivity and canonical coordinates. Such property of a hyperbolic metric was obtained by Fried in [13] (see also [34]). From Thereom 2.21, taking $z=[x, y]$, we have that: if $f: M \rightarrow M$ is expansive, has canonical coordinates and $\mathrm{d}$ is self-similar then for all $\varepsilon>0$ there is $\delta>0$ such that if $\mathrm{d}(x, y)<\delta$ then

$$
\begin{aligned}
& \mathrm{d}([x, y], x) \leq(1+\varepsilon) \mathrm{d}(x, y) \\
& \mathrm{d}([x, y], y) \leq(1+\varepsilon) \mathrm{d}(x, y)
\end{aligned}
$$

This property was previously proved by Dovbish in [8, Remark 1.5]. 


\section{Topological entropy}

Let $(M, \mathrm{~d})$ be a compact metric space. For $\varepsilon>0$ we say that

$$
\mathcal{U}=\left\{A_{1}, \ldots, A_{n}\right\}
$$

is an $(\varepsilon, \mathrm{d})$-cover of $X \subset M$ if $\cup_{i=1}^{n} A_{i}=X$ and $\operatorname{diam}_{\mathrm{d}}\left(A_{i}\right)<\varepsilon$ for all $i=$ $1, \ldots, n$. Define

$$
\operatorname{cov}_{\varepsilon}(X, \mathrm{~d})=\min \{\operatorname{card}(\mathcal{U}): \mathcal{U} \text { is an }(\varepsilon, \mathrm{d}) \text {-cover of } X\}
$$

and

$$
\mathrm{d}_{n}^{f}(x, y)=\max _{|k| \leq n} \mathrm{~d}\left(f^{k}(x), f^{k}(y)\right) .
$$

Remark 3.1. Note that if $f$ is expansive and $d$ is a self-similar metric with expansive constant $\xi$ and expanding factor $\lambda$ then

$$
\mathrm{d}_{n}^{f}(x, y)=\lambda^{n} \mathrm{~d}(x, y)
$$

if $\mathrm{d}(x, y) \leq \xi / \lambda^{n}$. Equation (26) also holds if $\mathrm{d}_{n}^{f}(x, y) \leq \xi$.

Proposition 3.2. If $f$ is expansive and $\mathrm{d}$ is a self-similar metric with expansive constant $\xi$ and expanding factor $\lambda$ then

$$
\operatorname{cov}_{\xi / \lambda^{k}}(X, \mathrm{~d})=\operatorname{cov}_{\xi}\left(X, \mathrm{~d}_{k}^{f}\right)
$$

for all $k \geq 0$ and $X \subset M$.

Proof. If $\mathcal{U}$ is an $\left(\xi / \lambda^{k}, \mathrm{~d}\right)$-cover of $X$ then $\operatorname{diam}_{\mathrm{d}}(U)<\xi / \lambda^{k}$ for all $U \in \mathcal{U}$. Equation (26) implies that $\operatorname{diam}_{\mathrm{d}_{k}^{f}}(U)<\xi$ and then $\mathcal{U}$ is an $\left(\xi, \mathrm{d}_{k}^{f}\right)$-cover of $X$. Then $\operatorname{cov}_{\xi / \lambda^{k}}(X, \mathrm{~d}) \geq \operatorname{cov}_{\xi}\left(X, \mathrm{~d}_{k}^{f}\right)$.

To prove the converse inequality consider $\mathcal{U}$ an $\left(\xi, \mathrm{d}_{k}^{f}\right)$-cover of $X$. Given $U \in \mathcal{U}$ we have that $\operatorname{diam}_{\mathrm{d}_{k}^{f}}(U)<\xi$, that is, $\operatorname{diam}\left(f^{j}(U)\right)<\xi$ if $|j| \leq k$. If $\operatorname{diam}(U) \geq \xi / \lambda^{k}$ then $\max \left\{\operatorname{diam}\left(f^{k}(U)\right), \operatorname{diam}\left(f^{-k}(U)\right)\right\} \geq \xi$ (which would be a contradiction). Then $\mathcal{U}$ is an $\left(\xi / \lambda^{k}, \mathrm{~d}\right)$-cover. This proves the other inequality.

It is known that the limit

$$
\mathrm{h}(X)=\lim _{n \rightarrow+\infty} \frac{1}{n} \log \left(\operatorname{cov}_{\xi}\left(X, \mathrm{~d}_{n}^{f}\right)\right)
$$

exists, is finite and independent of the expansive constant $\xi$ in the above expression, see for example [5]. This limit is the topological entropy of $X$ associated to $f$. The topological entropy of $f$ is defined as $\mathrm{h}(f)=\mathrm{h}(M)$.

Define

$$
\begin{array}{r}
\mathrm{d}_{n}^{+}(x, y)=\max _{0 \leq k \leq n} \mathrm{~d}\left(f^{k}(x), f^{k}(y)\right), \\
\mathrm{d}_{n}^{-}(x, y)=\max _{0 \leq k \leq n} \mathrm{~d}\left(f^{-k}(x), f^{-k}(y)\right) .
\end{array}
$$


Given $X \subset M$ define

$$
\begin{aligned}
\mathrm{h}^{+}(X) & =\lim _{n \rightarrow+\infty} \frac{1}{n} \log \left(\operatorname{cov} \xi\left(X, \mathrm{~d}_{n}^{+}\right)\right), \\
\mathrm{h}^{-}(X) & =\lim _{n \rightarrow+\infty} \frac{1}{n} \log \left(\operatorname{cov} \xi\left(X, \mathrm{~d}_{n}^{-}\right)\right) .
\end{aligned}
$$

These numbers depend on $f$.

Remark 3.3. The topological entropy considered in [10] (and in most texts, as for example in [5]) is ent $(f)=\mathrm{h}^{+}(M)$. It is easy to prove that

$$
2 \mathrm{~h}^{+}(M)=\mathrm{h}(f) \text {. }
$$

To avoid the introduction of a factor 2 in Equation (29) we defined the entropy as we did (which, again, is not the standard way).

Proposition 3.4. We have that

$$
\mathrm{h}^{+}(M)=\mathrm{h}^{-}(M)=\mathrm{h}(M) / 2 .
$$

Proof. Notice that $\mathcal{U}$ is an $\left(\xi, \mathrm{d}_{n}^{+}\right)$-cover of $M$ if and only if $f^{n}(\mathcal{U})$ is an $\left(\xi, \mathrm{d}_{n}^{-}\right)$cover of $M$. This proves that $\mathrm{h}^{+}(M)=\mathrm{h}^{-}(M)$.

Note that if $\mathcal{U}$ is an $\left(\xi, \mathrm{d}_{n}\right)$ cover of $M$ then $f^{-n}(\mathcal{U})$ is an $\left(\xi, \mathrm{d}_{2 n-1}^{+}\right)$cover. This implies that $\operatorname{cov}_{\xi}\left(M, \mathrm{~d}_{2 n-1}^{+}\right) \leq \operatorname{cov}_{\xi}\left(M, \mathrm{~d}_{n}\right)$ and $2 \mathrm{~h}^{+}(M) \leq \mathrm{h}(M)$. The converse inequality is analogous.

We say that a set $X \subset M$ is stable if $\operatorname{diam}\left(f^{n}(X)\right) \rightarrow 0$ as $n \rightarrow+\infty$. We say that it is unstable if $\operatorname{diam}\left(f^{n}(X)\right) \rightarrow 0$ as $n \rightarrow-\infty$. If $X$ is unstable and $\operatorname{diam}\left(f^{-n}(X)\right) \leq \xi$ for all $n \geq 0$ then

$$
\operatorname{cov}_{\xi / \lambda^{k}}(X, \mathrm{~d})=\operatorname{cov}_{\xi}\left(X, \mathrm{~d}_{k}^{+}\right)
$$

if the metric is self-similar.

\subsection{Canonical coordinates}

Let $f: M \rightarrow M$ be an expansive homeomorphism with canonical coordinates of a compact metric space. Recall the map [,] defined in Equation (1) giving the intersection of local stable and unstable sets. For $p \in M$ and $r>0$ small define

$$
C_{r}(p)=\left[W_{r}^{s}(p), W_{r}^{u}(p)\right]=\left\{[x, y]: x \in W_{r}^{s}(p), y \in W_{r}^{u}(p)\right\} .
$$

We will say that $C_{r}(p)$ is a product box around $p$. The sets $\left[\{x\}, W_{r}^{u}(p)\right]$ and $\left[W_{r}^{s}(p),\{y\}\right]$ will be called plaques of the product box.

We recall that the spectral decomposition theorem states that for an expansive homeomorphism $f$ with canonical coordinates the non-wandering set $\Omega(f)$ is a disjoint union $B_{1} \cup \cdots \cup B_{l}$ of compact $f$-invariant sets and each $f: B_{i} \rightarrow B_{i}$ is transitive. The sets $B_{i}$ are called basic sets. The spectral decomposition for hyperbolic diffeomorphisms of smooth manifolds is due to Smale. A proof in the topological setting can be found in [1, Theorem 3.4.4].

Assume that $3 \xi$ is an expansive constant and $\mathrm{d}$ is self-similar with expanding factor $\lambda$. 
Proposition 3.5. If $r<\xi$ and $C=C_{r}(p)$ is a product box then

$$
\mathrm{h}^{+}(C)=\mathrm{h}^{+}\left(P^{u}\right) \text { and } \mathrm{h}^{-}(C)=\mathrm{h}^{-}\left(P^{s}\right)
$$

for every unstable plaque $P^{u}$ and every stable plaque $P^{s}$ of $C$.

Proof. Let us only prove the first equality. Since $P^{u} \subset C$ we have that $\mathrm{h}^{+}\left(P^{u}\right) \leq$ $\mathrm{h}^{+}(C)$. Let $\pi: C \rightarrow P^{u}$ be the canonical projection. Consider $p, q \in C$. By the triangular inequality we have

$$
\begin{array}{ll}
\mathrm{d}\left(f^{n}(p), f^{n}(q)\right) \leq & \mathrm{d}\left(f^{n}(p), f^{n}(\pi(p))\right)+ \\
& \mathrm{d}\left(f^{n}(\pi(p)), f^{n}(\pi(q))\right)+\mathrm{d}\left(f^{n}(\pi(q)), f^{n}(q)\right)
\end{array}
$$

for all $n \in \mathbb{Z}$. If $n \geq 0$ then

$$
\begin{array}{ll}
\mathrm{d}\left(f^{n}(p), f^{n}(q)\right) \leq \quad & \lambda^{-n} \mathrm{~d}(p, \pi(p))+ \\
& \mathrm{d}\left(f^{n}(\pi(p)), f^{n}(\pi(q))\right)+\lambda^{-n} \mathrm{~d}(\pi(q), q)
\end{array}
$$

Thus

$$
\mathrm{d}\left(f^{n}(p), f^{n}(q)\right) \leq \mathrm{d}\left(f^{n}(\pi(p)), f^{n}(\pi(q))\right)+2 \xi / \lambda^{n} .
$$

Therefore, if $\mathrm{d}\left(f^{k}(\pi(p)), f^{k}(\pi(q))\right) \leq \xi$ for all $k=0,1, \ldots, n$ then

$$
\mathrm{d}\left(f^{k}(p), f^{k}(q)\right) \leq 3 \xi
$$

for all $k=0,1, \ldots, n$. That is, if $\mathrm{d}_{n}^{+}(\pi(p), \pi(q)) \leq \xi$ then $\mathrm{d}_{n}^{+}(p, q) \leq 3 \xi$. Consequently, if $\mathcal{U}$ is an $\left(\xi, \mathrm{d}_{n}^{+}\right)$-cover of $P^{u}$ then $\pi^{-1}(\mathcal{U})$ is a $\left(3 \xi, \mathrm{d}_{n}^{+}\right)$-cover of $C$. This implies that $\operatorname{cov}_{\xi}\left(P^{u}, \mathrm{~d}_{n}^{+}\right) \geq \operatorname{cov}_{3 \xi}\left(C, \mathrm{~d}_{n}^{+}\right)$and $\mathrm{h}^{+}\left(P^{u}\right) \geq \mathrm{h}^{+}(C)$.

Corollary 3.6. If $P_{1}^{u}$ and $P_{2}^{u}$ are unstable plaques of a common product box $C$ then $\mathrm{h}^{+}\left(P_{1}^{u}, \mathrm{~d}\right)=\mathrm{h}^{+}\left(P_{2}^{u}, \mathrm{~d}\right)$. Analogous for stable plaques.

Proof. By Proposition 3.5 we know that $\mathrm{h}^{+}\left(P_{i}^{u}\right)=\mathrm{h}^{+}(C)$.

\subsection{Homogeneous entropy}

We say that $f$ has homogeneous entropy if for all $x, y \in M$ it holds that $\mathrm{h}\left(W_{\xi}^{u}(x)\right)=\mathrm{h}\left(W_{\xi}^{u}(y)\right)$ and $\mathrm{h}\left(W_{\xi}^{s}(x)\right)=\mathrm{h}\left(W_{\xi}^{u}(y)\right)$.

Proposition 3.7. If $f$ has homogeneous entropy then $\mathrm{h}\left(W_{\xi}^{u}(x)\right)$ and $\mathrm{h}\left(W_{\xi}^{s}(x)\right)$ do not depend on the expansivity constant $\xi$.

Proof. It is direct from the definitions noting that $\mathrm{h}(X)=\mathrm{h}(f(X))$ for all $X \subset M$.

Proposition 3.8. If $f$ is expansive with canonical coordinates and homogeneous entropy then

$$
\mathrm{h}^{+}\left(W_{\xi}^{u}(x)\right)=\mathrm{h}^{-}\left(W_{\xi}^{s}(x)\right)=\frac{1}{2} \mathrm{~h}(M)
$$

for all $x \in M$. 
Proof. We will prove that $\mathrm{h}^{+}\left(W_{\varepsilon}^{u}(x)\right)=\frac{1}{2} \mathrm{~h}(M)$. Let $C_{1}, \ldots, C_{p}$ be a cover of $M$ by product boxes. By Proposition 3.4 we have that $\frac{1}{2} \mathrm{~h}(M)=\mathrm{h}^{+}(M)$. As in $[5$, Proposition 2.5.5] we can prove that

$$
\mathrm{h}^{+}(M)=\max _{i=1, \ldots, p} \mathrm{~h}^{+}\left(C_{i}\right) .
$$

Suppose that $\mathrm{h}^{+}(M)=\mathrm{h}^{+}\left(C_{j}\right)$ for some $j$. If $P^{u}$ is an unstable plaque of $C_{j}$, by Proposition 3.5 we know that $\mathrm{h}^{+}\left(C_{j}\right)=\mathrm{h}^{+}\left(P^{u}\right)$. This finishes the proof.

We say that a basic set $\Lambda \subset \Omega(f)$ is extremal if it is an attractor or a repeller of the spectral decomposition of the non-wandering set.

Proposition 3.9. An expansive homeomorphism with canonical coordinates has homogeneous entropy if and only if $\mathrm{h}(M)=\mathrm{h}(\Lambda)$ for every extremal basic set $\Lambda \subset \Omega(f)$.

Proof. To prove the direct part suppose that $\Lambda$ is an attractor. In this case we have that $W_{\xi}^{u}(x) \subset \Lambda$ for all $x \in \Lambda$. If we apply Proposition 3.8 to $f: M \rightarrow M$ and $f: \Lambda \rightarrow \Lambda$ we obtain

$$
\mathrm{h}(M)=2 \mathrm{~h}^{+}\left(W_{\xi}^{u}(x)\right)=\mathrm{h}(\Lambda)
$$

for all $x \in \Lambda$.

To prove the converse assume that $\mathrm{h}(M)=\mathrm{h}(\Lambda)$ for every extremal basic set $\Lambda \subset \Omega(f)$. Since $\mathrm{h}\left(\Lambda^{\prime}\right) \leq \mathrm{h}(M)$ for every basic set $\Lambda^{\prime}$ we have that $\mathrm{h}\left(\Lambda^{\prime}\right) \leq \mathrm{h}(\Lambda)$ for every extremal $\Lambda$. Given $x \in M$ there are $y \in B_{\xi}(x)$ and an attractor $\Lambda_{a}$ such that $\mathrm{d}\left(f^{n}(x), \Lambda_{a}\right) \rightarrow 0$ as $n \rightarrow+\infty$. Applying Corollary 3.6 we conclude that $\mathrm{h}^{+}\left(W_{\xi}^{u}(x)\right)=\mathrm{h}^{+}\left(W_{\xi}^{u}(z)\right)$ for $z \in \Lambda_{a}$.

Theorem 3.10. If $f: M \rightarrow M$ is a transitive expansive homeomorphism with canonical coordinates of a compact metric space $M$ then $f$ has homogeneous entropy.

Proof. Take $p, q \in M$ and consider $\varepsilon_{p}, \varepsilon_{q}>0$. Let $C_{p}, C_{q}$ be boxes around $p, q$ respectively such that $W_{\varepsilon_{p}}^{s}(p)$ is a plaque of $C_{p}$ and $W_{\varepsilon_{q}}^{s}(q)$ is a plaque of $C_{q}$. Take $x \in M$ with orbit dense in $M$. Assume that $x \in C_{p}$ and denote by $P_{x}$ the stable plaque of $x$ in $C_{p}$. Take $n \geq 0$ such that $f^{n}\left(P_{x}\right) \subset C_{q}$.

We have that $\mathrm{h}^{-}\left(P_{x}\right)=\mathrm{h}^{-}\left(f^{n}\left(P_{x}\right)\right)$. Let $Q_{x}$ be the stable plaque of $f^{n}(x)$ in $C_{q}$. Since $f^{n}\left(P_{x}\right) \subset Q_{x}$ we have that $\mathrm{h}^{-}\left(f^{n}\left(P_{x}\right)\right) \leq \mathrm{h}^{-}\left(Q_{x}\right)$. By Corollary 3.6 we have that $\mathrm{h}^{-}\left(Q_{x}\right)=\mathrm{h}^{-}\left(W_{\varepsilon_{q}}^{s}(q)\right)$. Then, we have proved that

$$
\mathrm{h}^{-}\left(W_{\varepsilon_{p}}^{s}(p)\right) \leq \mathrm{h}^{-}\left(W_{\varepsilon_{q}}^{s}(q)\right) .
$$

Analogously, we can prove the converse inequality and the proof ends. 


\subsection{Capacity}

Given $X \subset M$ the capacity is defined as

$$
\operatorname{cap}(X, \mathrm{~d})=\lim _{\varepsilon \rightarrow 0} \frac{\log \left(\operatorname{cov}_{\varepsilon}(X, \mathrm{~d})\right)}{-\log (\varepsilon)}
$$

whenever this limit exists. The following result is based on [10, Theorem 5.3] where an inequality is proved.

Theorem 3.11. If $f$ is expansive and $\mathrm{d}$ is a self-similar metric with expanding factor $\lambda$ then the limit (28) exists and

$$
\operatorname{cap}(X, \mathrm{~d})=\frac{\mathrm{h}(X)}{\log (\lambda)}
$$

for every $X \subseteq M$.

Proof. Given $\varepsilon>0$ small consider a positive integer $n(\varepsilon)$ such that

$$
\xi / \lambda^{n(\varepsilon)+1} \leq \varepsilon<\xi / \lambda^{n(\varepsilon)} .
$$

Then

$$
\operatorname{cov}_{\xi / \lambda^{n(\varepsilon)+1}}(X, \mathrm{~d}) \geq \operatorname{cov}_{\varepsilon}(X, \mathrm{~d}) \geq \operatorname{cov}_{\xi / \lambda^{n(\varepsilon)}}(X, \mathrm{~d})
$$

and

$$
\frac{\log \left(\operatorname{cov}_{\xi / \lambda^{n(\varepsilon)+1}}(X, \mathrm{~d})\right)}{-\log \left(\xi / \lambda^{n(\varepsilon)}\right)} \geq \frac{\log \left(\operatorname{cov}_{\varepsilon}(X, \mathrm{~d})\right)}{-\log (\varepsilon)} \geq \frac{\log \left(\operatorname{cov}_{\xi / \lambda^{n(\varepsilon)}}(X, \mathrm{~d})\right)}{-\log \left(\xi / \lambda^{n(\varepsilon)+1}\right)} .
$$

By Proposition 3.2 we have that $\operatorname{cov}_{\xi / \lambda^{k}}(X, \mathrm{~d})=\operatorname{cov}_{\xi}\left(X, \mathrm{~d}_{k}^{f}\right)$. Then

$$
\frac{\log \left(\operatorname{cov}_{\xi}\left(X, \mathrm{~d}_{n(\varepsilon)+1}^{f}\right)\right)}{n(\varepsilon) \log \lambda-\log (\xi)} \geq \frac{\log \left(\operatorname{cov}_{\varepsilon}(X, \mathrm{~d})\right)}{-\log (\varepsilon)} \geq \frac{\log \left(\operatorname{cov}_{\xi}\left(X, \mathrm{~d}_{n(\varepsilon)}^{f}\right)\right)}{(n(\varepsilon)+1) \log \lambda-\log (\xi)}
$$

Since $\lim _{\varepsilon \rightarrow 0} n(\varepsilon)=+\infty$ we conclude that

$$
\frac{\mathrm{h}(X)}{\log (\lambda)} \geq \operatorname{cap}(X, \mathrm{~d}) \geq \frac{\mathrm{h}(X)}{\log (\lambda)}
$$

which proves the result.

Proposition 3.12. Assume that $f: M \rightarrow M$ is an expansive homeomorphism with self-similar metric $\mathrm{d}$ and expanding factor $\lambda$. For every unstable set $X$ and every stable set $Y$ it holds that:

$$
\operatorname{cap}(X, \mathrm{~d})=\frac{\mathrm{h}^{+}(X)}{\log (\lambda)}=\frac{\mathrm{h}(X)}{\log (\lambda)}
$$

and

$$
\operatorname{cap}(Y, \mathrm{~d})=\frac{\mathrm{h}^{-}(Y)}{\log (\lambda)}=\frac{\mathrm{h}(Y)}{\log (\lambda)} .
$$


Proof. It is analogous to the proof of Theorem 3.11 using Equation (27).

In this case it is easy to see that $\mathrm{h}^{-}(X)=\mathrm{h}^{+}(Y)=0$.

Corollary 3.13. If $P_{1}^{u}$ and $P_{2}^{u}$ are unstable plaques of a common product box $C$ then $\operatorname{cap}\left(P_{1}^{u}, \mathrm{~d}\right)=\operatorname{cap}\left(P_{2}^{u}, \mathrm{~d}\right)$. Analogous for stable plaques.

Proof. It follows by Corollary 3.6 and Proposition 3.12.

\subsection{Expanding factors}

The purpose of this section is to study the set of expanding factors $\lambda$ of a self-similar metric that can be associated to a given expansive homeomorphism $f: M \rightarrow M$. Note that if there is a self-similar metric d with expanding factor $\lambda$ then the metric $\mathrm{d}^{\prime}(x, y)=[\mathrm{d}(x, y)]^{\alpha}, 0<\alpha<1$, is self-similar with expanding factor $\lambda^{\alpha}<\lambda$. This means that the set of expanding factors is an interval.

Let $\lambda_{\text {sup }}(f) \in(1,+\infty]$ be such that every $\lambda<\lambda_{\text {sup }}$ is the expanding factor of a self-similar metric for $f$, and every $\lambda>\lambda_{\text {sup }}$ is not.

Remark 3.14. The metric defined in Example 2.7 has expanding factor $\lambda_{\text {sup }}$.

The following result characterizes the systems with $\lambda_{\text {sup }}$ infinite. The topological dimension [19] of $M$ will be denote as $\operatorname{dim}(M)$. In [19] it is shown that $\operatorname{dim}(M) \leq \operatorname{cap}(M, \mathrm{~d})$ for every metric d.

Proposition 3.15. $\lambda_{\mathrm{sup}}=+\infty$ if and only if $M$ is totally disconnected.

Proof. Assume that $M$ is totally disconnected. From [20, Corollary 2.9] we known that $f$ is conjugate with a subshift. Recall that in Example 2.5 we gave a self-similar metric for the shift homeomorphism with an arbitrary expanding factor $\lambda>1$.

If $M$ is not totally disconnected then the topological dimension of $M$ is positive. Applying Theorem 3.11 we have

$$
\operatorname{dim}(M) \log (\lambda) \leq \mathrm{h}(f)
$$

for every expanding factor $\lambda$ of a hyperbolic self-similar metric. This implies

$$
\lambda \leq e^{\mathrm{h}(f) / \operatorname{dim}(M)}
$$

and $\lambda_{\text {sup }}$ is finite.

Remark 3.16. As noticed in [10], Equation (30) implies that: 1) if a compact metric space admits an expansive homeomorphism then its topological dimension is finite (a result first proved by Mañé [23]) and 2) if $\operatorname{dim}(M)>0$ then every expansive homeomorphism of $M$ has positive topological entropy.

In what follows assume that $\operatorname{dim}(M)>0$. Define the ideal expanding factor of $f$ as

$$
\lambda_{\text {ideal }}=e^{\mathrm{h}(f) / \operatorname{dim}(M)} .
$$

Obviously, if $\lambda$ is an expanding factor then $\lambda \leq \lambda_{\text {ideal }}$. 
Example 3.17 (Pseudo-Anosov maps again). On surfaces every expansive homeomorphism admits a self-similar metric with ideal expanding factor. This is because the metric that we constructed in Example 2.6 expands with the factor associated to the transverse measures. Since the topological entropy of a pseudo-Anosov map is $2 \log (\lambda)$ and the topological dimension of a surface is 2 , the stretching factor of a pseudo-Anosov diffeomorphism is $\lambda_{\text {ideal }}$.

It is of interest for the next result to remark that an expansive axiom A diffeomorphism may not be Anosov, for example there are quasi-Anosov diffeomorphisms that are not Anosov [12]. Quasi-Anosov diffeomorphisms are known to be axiom $\mathrm{A}$ and expansive.

Theorem 3.18. Let $f: M \rightarrow M$ be an expansive homeomorphism of a compact connected manifold with self-similar metric d. If $f: \Omega(f) \rightarrow \Omega(f)$ has ideal expanding factor then $\Omega(f)$ has non-empty interior. If in addition $f$ is an axiom A diffeomorphism then $f$ is a transitive Anosov diffeomorphism and the dimension of stable and unstable manifolds coincide.

Proof. It is known [2] (see also [17, Equation (3.3.1)]) that the topological entropy of $f$ restricted to the non-wandering set equals the topological entropy of $f$ in $M$. Since $f$ has ideal expanding factor on $\Omega(f)$ we can apply Theorem 3.11 to conclude that $\operatorname{dim}(\Omega(f))=\operatorname{dim}(M)$. From [19, Theorem IV 3] we know that $\Omega(f)$ has non-empty interior.

If $f$ is axiom A we can apply [11] to conclude that $\Omega(f)=M$ and that $f$ is a transitive Anosov. From Theorem 3.10, $f$ has homogeneous entropy. Applying Proposition 3.8 we have that

$$
\mathrm{h}^{+}\left(W_{\xi}^{u}(x)\right)=\mathrm{h}^{-}\left(W_{\xi}^{s}(x)\right)=\frac{1}{2} \mathrm{~h}(M)
$$

for all $x \in M$. By Proposition 3.12 we conclude that

$$
\operatorname{cap}\left(W_{\xi}^{u}(x), \mathrm{d}\right)=\operatorname{cap}\left(W_{\xi}^{s}(x), \mathrm{d}\right)=\frac{\operatorname{dim}(M)}{2} .
$$

If $\operatorname{dim}\left(W^{s}(x)\right) \neq \operatorname{dim}\left(W^{u}(x)\right)$ then one of these numbers is strictly greater than $\operatorname{dim}(M) / 2$. We arrive to a contradiction because the capacity is greater or equal than the dimension.

As a consequence, an Anosov diffeomorphism of a three dimensional manifold does not admit a self-similar metric with ideal expanding factor.

\section{Holonomy on canonical coordinates}

In this section we give some simple properties of pseudo isometries and the Hausdorff measure. Assuming the existence of canonical coordinates we show that holonomies are pseudo isometries, and consequently the Hausdorff measure is preserved by holonomy. We also show that on a Peano continuum the condition of isometric holonomies implies the transitivity of the homeomorphism. 


\subsection{Pseudo isometries and Hausdorff measure}

Given metric spaces $\left(X_{i}, \mathrm{~d}_{i}\right), i=1,2$ we say that a homeomorphism $h: X_{1} \rightarrow X_{2}$ is a pseudo isometry if for all $\varepsilon>0$ there is $\delta>0$ such that if $0<\mathrm{d}_{1}(x, y)<\delta$ then

$$
\left|\frac{\mathrm{d}_{2}(h(x), h(y))}{\mathrm{d}_{1}(x, y)}-1\right|<\varepsilon .
$$

In this case, $X_{1}$ and $X_{2}$ are said to be pseudo isometric.

Given a compact metric space $(X, \mathrm{~d})$ and $r>0$ define $C_{r}(X, \mathrm{~d})$ as the set of countable covers $\mathcal{U}$ of $X$ such that $\operatorname{diam}(U)<r$ for all $U \in \mathcal{U}$. For $d>0$ define

$$
\mu_{r}^{d}(X, \mathrm{~d})=\inf _{\mathcal{U} \in C_{r}} \sum_{U \in \mathcal{U}}(\operatorname{diam}(U))^{d}
$$

and

$$
\mu^{d}(X, \mathrm{~d})=\lim _{r \rightarrow 0} \mu_{r}^{d}(X, \mathrm{~d}) .
$$

Proposition 4.1. Let $h:\left(X_{1}, \mathrm{~d}_{1}\right) \rightarrow\left(X_{2}, \mathrm{~d}_{2}\right)$ be a pseudo isometry of compact metric spaces. Then $\mu^{d}\left(X_{1}, \mathrm{~d}_{1}\right)=\mu^{d}\left(X_{2}, \mathrm{~d}_{2}\right)$ for all $d>0$.

Proof. Given $\varepsilon>0$ take $\delta>0$ such that if $x, y \in X_{1}$ and $\mathrm{d}_{1}(x, y)<\delta$ then

$$
\left|\frac{\mathrm{d}_{2}(h(x), h(y))}{\mathrm{d}_{1}(x, y)}-1\right|<\varepsilon .
$$

That is

$$
(1-\varepsilon) \mathrm{d}_{1}(x, y)<\mathrm{d}_{2}(h(x), h(y))<(1+\varepsilon) \mathrm{d}_{1}(x, y) .
$$

Therefore, if $\operatorname{diam}_{1}(U)<\delta$ then $\operatorname{diam}_{2}(h(U))<(1+\varepsilon) \operatorname{diam}_{1}(U)$. If $r<\delta$ and $\mathcal{U} \in C_{r}\left(X_{1}, \mathrm{~d}_{1}\right)$ then $h(\mathcal{U}) \in C_{r(1+\varepsilon)}\left(X_{2}, \mathrm{~d}_{2}\right)$. Then, for all $\mathcal{U} \in C_{r}\left(X_{1}, \mathrm{~d}_{1}\right)$ it holds that

$$
\sum_{U \in \mathcal{U}} \operatorname{diam}_{2}(h(U))^{d}<(1+\varepsilon)^{d} \sum_{U \in \mathcal{U}} \operatorname{diam}_{1}(U)^{d}
$$

This implies that

$$
\mu_{r(1+\varepsilon)}^{d}\left(X_{2}, \mathrm{~d}_{2}\right) \leq(1+\varepsilon)^{d} \mu_{r}^{d}\left(X_{1}, \mathrm{~d}_{1}\right),
$$

and

$$
\mu^{d}\left(X_{2}, \mathrm{~d}_{2}\right) \leq(1+\varepsilon)^{d} \mu^{d}\left(X_{1}, \mathrm{~d}_{1}\right) .
$$

Since this holds for all $\varepsilon>0$ we conclude that $\mu^{d}\left(X_{2}, \mathrm{~d}_{2}\right) \leq \mu^{d}\left(X_{1}, \mathrm{~d}_{1}\right)$. The other inequality is analogous.

\subsection{Local form of the metric and transitivity}

Fix a product box $C$ and $p \in C$. For $x \in C$ define its coordinates relative to $p$ as

$$
\begin{aligned}
& x^{s}=W_{\xi}^{s}(p) \cap W_{\xi}^{u}(x), \\
& x^{u}=W_{\xi}^{u}(p) \cap W_{\xi}^{s}(x) .
\end{aligned}
$$


Define a metric $\mathrm{d}_{p}$ on $C$ by

$$
\mathrm{d}_{p}(x, y)=\max \left\{\mathrm{d}\left(x^{s}, y^{s}\right), \mathrm{d}\left(x^{u}, y^{u}\right)\right\}
$$

for all $x, y \in C$.

Lemma 4.2. If $|1-x|<\sigma<1$ then

$$
\left|1-\frac{1}{x}\right|<\frac{\sigma}{1-\sigma}
$$

Proof. We have that $1-x<\sigma<1$. Then $0<1-\sigma<x$.

Lemma 4.3. If $f: M \rightarrow M$ is expansive and $\mathrm{d}$ is self-similar then the holonomy map on a product box $C \subset M$ is a pseudo isometry.

Proof. Taking positive iterates of $C$ we can assume that stable plaques of $C$ have diameter smaller than the expansivity constant $\xi$. Denote by $\pi: P_{1}^{u} \rightarrow P_{2}^{u}$ the holonomy of two unstable plaques of $C$. Fix $p, q \in P_{1}^{u}$. By the triangular inequality of the metric we have that

$$
\begin{aligned}
\left|\mathrm{d}\left(f^{n}(p), f^{n}(q)\right)-\mathrm{d}\left(f^{n}(\pi(p)), f^{n}(\pi(q))\right)\right| \leq & \mathrm{d}\left(f^{n}(p), f^{n}(\pi(p))\right)+ \\
& \mathrm{d}\left(f^{n}(\pi(q)), f^{n}(q)\right)
\end{aligned}
$$

for all $n \in \mathbb{Z}$. If $n \geq 0$ then

$$
\left|\mathrm{d}\left(f^{n}(p), f^{n}(q)\right)-\mathrm{d}\left(f^{n}(\pi(p)), f^{n}(\pi(q))\right)\right| \leq \frac{\mathrm{d}(p, \pi(p))+\mathrm{d}(\pi(q), q)}{\lambda^{n}}
$$

because $p, \pi(p)$ and $q, \pi(q)$ are in stable plaques and d is self-similar with expanding factor $\lambda>1$. If $\mathrm{d}(p, \pi(p))<\xi$ and $\mathrm{d}(\pi(q), q)<\xi$ then

$$
\left|\mathrm{d}\left(f^{n}(p), f^{n}(q)\right)-\mathrm{d}\left(f^{n}(\pi(p)), f^{n}(\pi(q))\right)\right| \leq \frac{2 \xi}{\lambda^{n}} .
$$

Take $m \geq 0$ such that

$$
\xi / \lambda^{m+1}<\max \{\mathrm{d}(p, q), \mathrm{d}(\pi(p), \pi(q))\} \leq \xi / \lambda^{m} .
$$

Then

$$
\begin{aligned}
|\mathrm{d}(p, q)-\mathrm{d}(\pi(p), \pi(q))| & =\frac{1}{\lambda^{m}}\left|\mathrm{~d}\left(f^{m}(p), f^{m}(q)\right)-\mathrm{d}\left(f^{m}(\pi(p)), f^{m}(\pi(q))\right)\right| \\
& \leq \frac{1}{\lambda^{m}} \cdot \frac{2 \xi}{\lambda^{m}}=\frac{2 \xi}{\lambda^{2 m}} .
\end{aligned}
$$

Given that $\xi / \lambda^{m+1}<\max \{\mathrm{d}(p, q), \mathrm{d}(\pi(p), \pi(q))\}$ we have

$$
|\mathrm{d}(p, q)-\mathrm{d}(\pi(p), \pi(q))| \leq \frac{2 \max \{\mathrm{d}(p, q), \mathrm{d}(\pi(p), \pi(q))\}}{\lambda^{m-1}} .
$$

Applying (31) we conclude that

$$
\left|\frac{\mathrm{d}(\pi(p), \pi(q))}{\mathrm{d}(p, q)}-1\right| \leq \frac{2}{\lambda^{m-1}-2} .
$$


Theorem 4.4. If $f: M \rightarrow M$ is expansive with $\mathrm{d}$ self-similar, $C \subset M$ is a product box and $p \in C$ then $(C, \mathrm{~d})$ and $\left(C, \mathrm{~d}_{p}\right)$ are pseudo isometric.

Proof. It follows by Lemma 4.3 and Theorem 2.21.

Remark 4.5. There are transitive expansive homeomorphisms with canonical coordinates for which the holonomy is not an isometry. Consider the classical derived from Anosov diffeomorphism on the two-dimensional torus [37]. Its non-wandering set consists of a fixed point and a basic set $\Omega$ that is locally the product of a Cantor set with an arc. Let $\gamma$ be a circle embedded in the torus and transverse to the arcs of $\Omega$. We have that $\Omega \cap \gamma$ is a Cantor set. Following the lines of $\Omega$ we can define a first return map $g: \Omega \cap \gamma \rightarrow \Omega \cap \gamma$. If $p, q \in \gamma$ are end points of a $g a p$ then $\mathrm{d}\left(g^{n}(p), g^{n}(q)\right) \rightarrow 0$ as $n \rightarrow \pm \infty$. This proves that, independently of the metric (self-similar or not), holonomies are not isometries.

We remark that the following result can be applied to Anosov diffeomorphisms of compact (connected) manifolds.

Theorem 4.6. Let $f$ be an expansive homeomorphism with canonical coordinates of a Peano continuum $M$. If $\mathrm{d}$ is self-similar and holonomies are isometries then $f$ is transitive.

Proof. Arguing by contradiction, consider from the spectral decomposition a repeller $R \subset \Omega(f)$, an attractor $A \subset \Omega(f)$ and a wandering point $x \in M$ such that $f^{n}(x) \rightarrow A$ and $f^{-n}(x) \rightarrow R$ as $n \rightarrow+\infty$. From [27, Theorem 8.25] we know that Peano continua are locally arc connected. Since we have local product structure we have that stable and unstable plaques are locally arc connected. Then, there are $N>0, y \in R$ and an $\operatorname{arc} l \subset W_{\xi}^{u}(y)$ from $y$ to $f^{-N}(x)$.

Consider $\delta>0$ such that if $\mathrm{d}(p, q) \leq 2 \delta$ then $W_{\xi}^{s}(p) \cap W_{\xi}^{u}(q) \neq \emptyset$. Take $z$ in the attractor $A$ such that $f^{N}(x) \in W_{\delta}^{s}(z)$. Let $\gamma=f^{2 N}(l)$, an arc from $f^{N}(x)$ to $f^{2 N}(y) \in R$ contained in the (global) unstable set of $f^{N}(x)$. Consider $\gamma$ ordered from $f^{N}(x)$ to $f^{2 N}(y)$ and take $p_{0}=f^{N}(x)<p_{1}<\cdots<p_{k}=f^{2 N}(y)$ points in $\gamma$ such that $\mathrm{d}\left(p_{i-1}, p_{i}\right)<\delta$ for each $i=1, \ldots, k$. Since $f^{N}(x) \in W_{\delta}^{s}(z)$ we have that $\mathrm{d}\left(p_{0}, z\right)<\delta$ and then $\mathrm{d}\left(p_{1}, z\right)<2 \delta$. Then, we can define $q_{1}=$ $W_{\xi}^{u}(z) \cap W_{\xi}^{s}\left(p_{1}\right)$. Since the holonomy is an isometry we have that $\mathrm{d}\left(p_{1}, q_{1}\right)=$ $\mathrm{d}\left(f^{N}(x), z\right)<\delta$. Then, $\mathrm{d}\left(p_{2}, q_{1}\right)<2 \delta$ and we can define $q_{2}=W_{\xi}^{u}\left(q_{1}\right) \cap W_{\xi}^{s}\left(p_{2}\right)$. Inductively we obtain a sequence $q_{1}, \ldots, q_{k}$ as $q_{i+1}=W_{\xi}^{u}\left(q_{i}\right) \cap W_{\xi}^{s}\left(p_{i+1}\right)$. The point $q_{k}$ is in $W^{u}(z) \cap W^{s}\left(f^{2 N}(y)\right)$. That is, $q_{k} \in A \cap R$. This proves the transitivity of $f$.

Let us explain why Theorem 4.6 is not true if we do not assume that $M$ is locally connected. We will consider a subshift of finite type on 4 symbols. The transition matrix

$$
\left(\begin{array}{llll}
1 & 1 & 1 & 1 \\
1 & 1 & 1 & 1 \\
0 & 0 & 1 & 1 \\
0 & 0 & 1 & 1
\end{array}\right)
$$


defines a subshift of finite type $f$ on a Cantor set $M$. From [38] we know that subshifts of finite type have canonical coordinates (or equivalently, the shadowing property). It is clear that $f$ is not transitive because the non-wandering set consists on a repeller and an attractor. Also, we can consider a self-similar metric with isometric holonomy (the metric given in Example 2.5).

\section{The intrinsic measure}

In $§ 5.2$ we will apply our results to the construction of the intrinsic measure of a topologically mixing expansive homeomorphism with canonical coordinates. In $§ 5.1$ we recall some known facts from $[1,29]$ that we need.

\subsection{Geometric constructions and Markov partitions}

Let $\mathbb{N}$ be the set of non-negative integers and consider a finite set $\mathcal{U}$. Let $Q \subset \mathcal{U}^{\mathbb{N}}$ be a topologically mixing subshift of finite type.

Let $(X, \rho)$ be a compact metric space and denote by $2^{X}$ the set of compact subsets of $X$. Consider $\Delta: Q \times \mathbb{N} \rightarrow 2^{X}$ and $K_{*}, K^{*}>0$ such that

1. $\Delta(\omega, n+1) \subset \Delta(\omega, n)$,

2. for each $(\omega, n) \in Q \times \mathbb{N}$ there are balls $B_{*}(\omega, n)$ and $B^{*}(\omega, n)$ of radius $K_{*} / \lambda^{n}$ and $K^{*} / \lambda^{n}$ respectively, such that

$$
B_{*}(\omega, n) \subset \Delta(\omega, n) \subset B^{*}(\omega, n),
$$

3. $\operatorname{int}\left(B_{*}\left(\omega_{1}, n\right)\right) \cap \operatorname{int}\left(B_{*}\left(\omega_{2}, n\right)\right)=\emptyset$ if $\left.\omega_{1}\right|_{\{0,1, \ldots, n\}} \neq\left.\omega_{2}\right|_{\{0,1, \ldots, n\}}$,

where $\operatorname{int}(A)$ denotes the interior of $A$. Define

$$
F_{\Delta}=\cap_{n \geq 0} \cup_{\omega \in Q} \Delta(\omega, n) \subset X .
$$

Theorem 5.1. $[28,29]$ In the above conditions, if $d=\operatorname{cap}\left(F_{\Delta}, \rho\right)$ then

$$
0<\mu^{d}\left(F_{\Delta}, \rho\right)<\infty .
$$

Proof. See [29] Theorems 13.1 and 13.4.

Let $f: M \rightarrow M$ be an expansive homeomorphism with canonical coordinates. A closed subset $R \subset M$ is a rectangle if $\operatorname{diam}(C)<\delta, R$ is the closure of its interior and $[x, y] \in R$ for all $x, y \in R$. Given $x \in R$ denote by $R^{u}(x)=\{y \in$ $R:[y, x]=y\}$. Assume that the diameter of the rectangle is so small that $R^{u}(x) \subset W_{\xi}^{u}(x)$ for all $x \in R$.

Proposition 5.2. For every $x \in \operatorname{int}(R)$ there is $r>0$ such that for all $y \in$ $R^{s}(x)$ it holds that

$$
W_{r}^{u}(y) \subset R^{u}(y)
$$

Proof. It follows by the compactness of $R$ and the product structure. 
A finite cover of $M$ by rectangles $\mathcal{U}=\left\{R_{1}, \ldots, R_{p}\right\}$ of $M$ is a Markov partition for $f: M \rightarrow M$ if

1. $\operatorname{int}\left(R_{i}\right) \cap \operatorname{int}\left(R_{j}\right)=\emptyset$ if $i \neq j$,

2. for each $x \in \operatorname{int}\left(R_{i}\right) \cap f^{-1}\left(\operatorname{int}\left(R_{j}\right)\right)$ we have $f\left(R_{i}^{s}(x)\right) \subset R_{j}^{s}(f(x))$ and $R_{j}^{u}(f(x)) \subset f\left(R_{i}^{u}(x)\right)$.

Every expansive homeomorphism with canonical coordinates admits Markov partitions by rectangles of arbitrarily small diameter, see [1, Theorem 4.2.8]. Define

$$
Q=\left\{\omega \in \mathcal{U}^{\mathbb{N}}: \operatorname{int}(\omega(j)) \cap f^{-1}(\operatorname{int}(\omega(j+1))) \neq \emptyset \text { for all } j \geq 0\right\} .
$$

If $f$ is topologically mixing we can apply [1, Theorem 4.3.5] to obtain that $Q$ is a topologically mixing subshift of finite type. For each rectangle $R_{i} \in \mathcal{U}$ fix an unstable plaque $P_{R_{i}}^{u} \subset R_{i}$ and define

$$
X=\cup_{i=1}^{p} P_{R_{i}}^{u} .
$$

Given $(\omega, n) \in Q \times \mathbb{N}$ define $\Delta(\omega, n)=X \cap\left(\cap_{j=0}^{n} f^{-j}(\omega(j))\right)$.

Remark 5.3. A point $x \in X$ is in $\Delta(\omega, n)$ if $f^{j}(x)$ is in the rectangle $\omega(j) \in \mathcal{U}$ for $j=0, \ldots, n$. Since $\mathcal{U}$ is a cover of $M$ we conclude that the set $F_{\Delta}$ defined in (32) coincides with $X$.

Proposition 5.4. With the previous notation, it holds that $\Delta(\omega, n) \subset W_{\xi / \lambda^{n}}^{u}(x)$ for all $x \in \Delta(\omega, n)$.

Proof. For all $y \in \Delta(\omega, n)$ we know that $f^{n}(y) \in \omega(n)$. Denote by $R_{n}$ the rectangle $\omega(n) \in \mathcal{U}$. Since $R_{n}^{u}\left(f^{n}(y)\right) \subset W_{\xi}^{u}\left(f^{n}(x)\right)$ we have that $f^{n}(y) \in$ $W_{\xi}^{u}\left(f^{n}(x)\right)$. Then, $y \in f^{-n}\left(W_{\xi}^{u}\left(f^{n}(x)\right)\right)=W_{\xi / \lambda^{n}}^{u}(x)$.

The following result is based on [29, Theorem 22.1].

Theorem 5.5. Let $f: \Lambda \rightarrow \Lambda$ be a topologically mixing expansive homeomorphism with canonical coordinates of a compact metric space $\Lambda$ with a self-similar metric $\rho$. If $d=\operatorname{cap}\left(W_{\xi}^{u}(x), \rho\right)$ then

$$
0<\mu^{d}\left(W_{\xi}^{u}(x), \rho\right)<\infty
$$

for all $x \in \Lambda$.

Proof. For each rectangle $R \in \mathcal{U}$ fix an interior point $x_{R}$. Consider $r_{R}>0$ from Proposition 5.2. Define $K_{*}=\min \left\{r_{R}: R \in \mathcal{U}\right\}$ and $K^{*}=\xi$. From Theorem 5.1 we have that $0<\mu^{d}(X, \rho)<\infty$, where $X$ is given by (33). This is because $F_{\Delta}=X$ (Remark 5.3). Since $f$ is topologically mixing we conclude (34). 


\subsection{Intrinsic ergodicity}

Let $f: M \rightarrow M$ be an expansive homeomorphism with canonical coordinates of a compact metric space. In addition assume that $f$ is topologically mixing. In [3] Bowen (see also [1, Theorem 11.5.13]) proved that topologically mixing expansive homeomorphisms with canonical coordinates have the specification property. And in [4] he proved that expansive homeomorphisms with specification (on a compact metric space) admit a unique measure with maximal entropy. A homeomorphism with a unique invariant measure maximizing the entropy is called intrinsically ergodic [39]. The purpose of this section is to show that this special measure can be naturally constructed using a self-similar metric.

Assume that $\mathrm{d}$ is self-similar with expanding factor $\lambda>1$ and define

$$
d=\frac{\mathrm{h}(f)}{2 \log (\lambda)} .
$$

Let $\mu$ be the Borel measure on $M$ such that given a rectangle $P^{s} \times P^{u} \subset M$ it holds that

$$
\mu\left(P^{s} \times P^{u}\right)=\mu^{d}\left(P^{s}\right) \mu^{d}\left(P^{s}\right)
$$

where $\mu^{d}$ is the $d$-dimensional Hausdorff measure. From Lemma 4.3 we know that the holonomy on a product box is a pseudo-isometry and Proposition 4.1 proves that pseudo-isometries preserve the Hausdorff measure. This means that the measure of a box does not depend on the plaques used to define the box.

Theorem 5.6. If $f$ is a topologically mixing expansive homeomorphism with canonical coordinates of a compact metric space then $\mu$ is the measure of maximal entropy. In particular, $\mu$ does not depend on the self-similar metric.

Proof. By Theorem 5.5, we have that

$$
0<\mu^{d}\left(W_{\varepsilon}^{s}(x)\right)<\infty \text { and } 0<\mu^{d}\left(W_{\varepsilon}^{u}(x)\right)<\infty
$$

for all $x \in M$ and for all $\varepsilon>0$. Given that $\mathrm{d}$ is self-similar we can apply $[9$, Scaling property 2.1] to conclude that

$$
\begin{aligned}
& \mu^{d}\left(f\left(W_{\xi}^{u}(x)\right), \mathrm{d}\right)=\lambda^{d} \mu^{d}\left(W_{\xi}^{u}(x), \mathrm{d}\right) \\
& \mu^{d}\left(f\left(W_{\xi}^{s}(x)\right), \mathrm{d}\right)=\lambda^{-d} \mu^{d}\left(W_{\xi}^{s}(x), \mathrm{d}\right)
\end{aligned}
$$

for all $d>0$. This implies that $\mu$ is $f$-invariant.

To prove that $\mu$ is the intrinsic measure we apply a result of Bowen explained in [7]. For this purpose we recall that $\mu$ is $f$-homogeneous if for all $\varepsilon>0$ there are $\delta>0$ and $c>0$ such that

$$
\mu\left(D_{\delta}^{n}(y)\right) \leq c \mu\left(D_{\varepsilon}^{n}(x)\right)
$$

for all $n \geq 0$ and all $x, y \in M$, where

$$
D_{\varepsilon}^{n}(x)=\left\{z \in M: \mathrm{d}\left(f^{i}(x), f^{i}(z)\right) \leq \varepsilon \text { if } 0 \leq i \leq n-1\right\} .
$$


According to [7, Proposition 19.7], in order to prove that $\mu$ maximizes the entropy it is sufficient to show that $\mu$ is $f$-homogeneous.

From Theorem 4.4 we know that for $\varepsilon>0$ there is $\delta>0$ such that if $\mathrm{d}(x, y)<\delta$ then

$$
(1-\varepsilon) \mathrm{d}_{x}(x, y)<\mathrm{d}(x, y)<(1+\varepsilon) \mathrm{d}_{x}(x, y) .
$$

If we define

$$
C_{\delta}^{n}(x)=\left[W_{\delta}^{s}(x), W_{\delta / \lambda^{n}}^{u}(x)\right]
$$

then

$$
C_{\delta /(1+\varepsilon)}^{n}(x) \subset D_{\delta}^{n}(x) \subset C_{\delta /(1-\varepsilon)}^{n}(x) .
$$

Define

$$
m^{\sigma}(\varepsilon)=\inf \left\{\mu^{d}\left(W_{\varepsilon}^{\sigma}(x)\right): x \in M\right\}
$$

and

$$
M^{\sigma}(\varepsilon)=\sup \left\{\mu^{d}\left(W_{\varepsilon}^{\sigma}(x)\right): x \in M\right\}
$$

for $\sigma=s, u$. We will show that $0<m^{\sigma}(\varepsilon) \leq M^{\sigma}(\varepsilon)<\infty$. From Theorem 5.5 we know that each $\mu^{d}\left(W_{\varepsilon}^{\sigma}(x)\right)$ is positive and finite. Since $f$ is transitive, we can take $z \in M$ with dense positive orbit. For $r>0$ given take $N$ large such that $\left\{z, f(z), \ldots, f^{N}(z)\right\}$ is $r$-dense in $M$. Then, it is easy to see that $m^{s}(\varepsilon)>\mu^{d}\left(W_{\varepsilon}^{s}(z)\right) \lambda^{-N}>0$. The other inequalities are analogous. From these inequalities, Equation (35) and the definition of $\mu$ we have

$m^{s}(\delta /(1+\varepsilon)) m^{u}(\delta /(1+\varepsilon)) \lambda^{-n} \leq \mu\left(D_{\delta}^{n}(x)\right) \leq M^{s}(\delta /(1-\varepsilon)) M^{u}(\delta /(1-\varepsilon)) \lambda^{-n}$

for all $x \in M$ and all $n \geq 0$. If we define

$$
c=\frac{M^{s}(\delta /(1-\varepsilon)) M^{u}(\delta /(1-\varepsilon))}{m^{s}(\delta /(1+\varepsilon)) m^{u}(\delta /(1+\varepsilon))}
$$

we obtain

$$
\mu\left(D_{\delta}^{n}(y)\right) \leq c \mu\left(D_{\delta}^{n}(x)\right)
$$

for all $x, y \in M$ and all $n \geq 0$. This proves that $\mu$ is $f$-homogeneous and as we explained the proof ends.

Remark 5.7. If we can apply [9, Corollary 7.4] then the Hausdorff dimension of $M$ is $\frac{\mathrm{h}(f)}{\log (\lambda)}$ (assuming the hypothesis of Theorem 5.6). The problem is that in [9] a global hypothesis (of the book) is that $M$ must be contained in Euclidean $\mathbb{R}^{n}$ (i.e., the metric of $M$ must be induced by an embedding of $M$ in some $\mathbb{R}^{n}$ ). Whether this hypothesis is essential or not is not clear to the author. Note that the finite dimensionality of a compact metric space admiting an expansive homeomorphism is proved in [23]. This and results from [19] implies that the space admits a topological embedding in $\mathbb{R}^{n}$. 


\section{References}

[1] N. Aoki and K. Hiraide, Topological theory of dynamical systems, North-Holland, 1994.

[2] R. Bowen, Topological entropy and Axiom A, Proc. Sympos. Pure Math. 14 (1970), $23-42$.

[3] _ Periodic points and measures for Axiom A diffeomorphisms, Trans. Amer. Math. Soc. 154 (1971), 377-397.

[4] , Some systems with unique equilibrium states, Math. Systems Theory 8 (1975), 193-202.

[5] M. Brin and G. Stuck, Introduction to Dynamical Systems, Cambridge University Press, 2003.

[6] E. M. Coven and W. L. Reddy, Positively expansive maps of compact manifolds, Springer, Lecture Notes in Math. 819 (1980), 96-110.

[7] M. Denker, C. Grillenberger, and K. Sigmund, Ergodic Theory on Compact Spaces, Vol. 527, Springer, Lecture notes in Math., 1976.

[8] S. A. Dovbysh, Optimal Lyapunov metrics of expansive homeomorphisms, Izvestiya: Mathematics 70 (2006), 883-929.

[9] K. Falconer, Fractal Geometry, John Wiley \& Sons, 1990.

[10] A. Fathi, Expansivity, hyperbolicity and Hausdorff dimension, Commun. Math. Phys. 126 (1989), 249-262.

[11] T. Fisher, Hyperbolic sets with non-empty interior, Disc. Cont. Dynam. Sys 15 (2006), 433-446.

[12] J. Franks and C. Robinson, A quasi-Anosov diffeomorphism that is not Anosov, Trans. of the AMS 223 (1976), 267-278.

[13] D. Fried, Métriques naturelles sur les espaces de Smale, C. R. Acad. Sci. Paris Sér. I Math. 297 (1983), 77-79.

$[14] \_$, Finitely presented dynamical systems, Ergod. Th. Dynam. Sys. 7 (1987), 489507.

[15] C. Fujita, H. Kato, and M. Matsumoto, Fractal metrics of Ruelle expanding maps and expanding ratios, Topology and its applications 157 (2010), 615-628.

[16] U. Hamenstadt, A new description of the Bowen-Margulis measure, Ergod. Th. \& Dynam. Sys. 9 (1989), 455-464.

[17] B. Hasselblatt and A. Katok, Introduction to the Modern Theory of Dynamical Systems, Cambridge University Press, 1995.

[18] K. Hiraide, Expansive homeomorphisms of compact surfaces are pseudo-Anosov, Osaka J. Math. 27 (1990), 117-162.

[19] W. Hurewicz and H. Wallman, Dimension Theory, Princeton Univ. Press, 1948.

[20] H. Keynes and J. Robertson, Generators for topological entropy and expansivity, Mathematical systems theory $\mathbf{3}$ (1969), 51-59.

[21] J. Lewowicz, Lyapunov Functions and Topological Stability, J. Diff. Eq. 38 (1980), 192209.

[22] _ Expansive homeomorphisms of surfaces, Bol. Soc. Bras. Mat. 20 (1989), 113-133.

[23] R. Mañé, Expansive homeomorphisms and topological dimension, Trans. of the AMS 252 (1979), 313-319.

[24] Ergodic theory and differentiable dynamics, Springer, 1983.

[25] G. A. Margulis, Certain measures associated with U-flows on compact manifolds, Functional Analysis and Its Applications 4 (1970), 55-67. 
[26] J. L. Massera, On Liapunoff's Conditions of Stability, Ann. of Math. 50 (1949), no. 3, $705-721$.

[27] S. Nadler Jr., Continuum Theory, Pure and Applied Mathematics, vol. 158, Marcel Dekker, New York, 1992.

[28] Y. Pesin and H. Weiss, On the Dimension of Deterministic and Random Cantor-like Sets, Symbolic Dynamics, and the Eckmann-Ruelle Conjecture, Commun. Math. Phys. 182 (1996), 105-153.

[29] Y. Pesin, Dimension Theory in Dynamical Systems, University of Chicago, 1997.

[30] W. L. Reddy, Expanding maps on compact metric spaces, Topology and its applications 13 (1982), 327-334.

[31] _ Expansive canonical coordinates are hyperbolic, Topology and its applications 15 (1983), 205-210.

[32] D. Ruelle, Thermodynamic Formalism, 2nd ed., Cambridge University Press, 2004.

[33] K. Sakai, Hyperbolic metrics of expansive homeomorphisms, Topology and its applications 63 (1995), 263-266.

[34] _ Shadowing properties of L-hyperbolic homeomorphisms, Topology and its Applications 112 (2001), 229-243.

[35] $\longrightarrow$ Various shadowing properties for positively expansive maps, Topology Appl. 131 (2003), 15-31.

[36] Ya. G. Sinai, Markov partitions and C-diffeomorphisms, Functional Analysis and Its Applications 2 (1968), 61-82.

[37] S. Smale, Differentiable dynamical systems, Bull. Amer. Math. Soc. 73 (1967), 747-817.

[38] P. Walters, On the pseudo orbit tracing property and its relationship to stability, Lect. Not. in Math. Springer 668 (1978), 231-244.

[39] B. Weiss, Intrinsically ergodic systems, Bull. Amer. Math. Soc. 76 (1970), 1226-1269. 